\title{
Power transmission and particle acceleration along the Io flux tube
}

\author{
S. L. G. Hess, ${ }^{1}$ P. Delamere, ${ }^{1}$ V. Dols, ${ }^{1}$ B. Bonfond, ${ }^{2}$ and D. Swift ${ }^{3}$ \\ Received 24 September 2009; revised 9 December 2009; accepted 4 January 2010; published 16 June 2010.
}

[1] Io's motion relative to the Jovian magnetic field generates a power of about $10^{12} \mathrm{~W}$, which is thought to propagate as an Alfvén wave along the magnetic field line. This power is transmitted to the electrons, which will then precipitate and generate the observed auroral phenomena from UV to radio wavelengths. A more detailed look at this hypothesis shows some difficulties: Can the Alfvén waves escape the torus or are they trapped inside? Where and how are the particles accelerated? In which direction? Is there enough power transmitted to the particles to explain the strong brightness of the auroral emissions in UV, IR, visible, and radio? In other words, can we make a global, consistent model of the Io-Jupiter interaction that matches all the observations? To answer these questions, we review the models and studies that have been proposed so far. We show that the Alfvén waves need to be filamented by a turbulent cascade process and accelerate the electrons at high latitude in order to explain the observations and to form a consistent scheme of the Io-Jupiter interaction.

Citation: Hess, S. L. G., P. Delamere, V. Dols, B. Bonfond, and D. Swift (2010), Power transmission and particle acceleration along the Io flux tube, J. Geophys. Res., 115, A06205, doi:10.1029/2009JA014928.

\section{Introduction}

[2] Io is the innermost Galilean satellite of Jupiter, orbiting at 5.95 Jovian radii from the planet center, deep inside the Jovian magnetosphere. It is constantly perturbed by the gravitational perturbations induced by the other Galilean satellites. As a result it is the most volcanically active body of the solar system. This volcanism generates a thin ionosphere at Io and injects $\sim 1$ ton/s of neutral gas in the Jovian magnetosphere, which is then ionized and dragged by the Jovian magnetic field, ultimately generating a dense plasma torus orbiting in the centrifugal equator, tilted by $\sim 7$ relative to the jovigraphic equator in which Io orbits. Io's latitude relative to the torus varies with the Io magnetic longitude. Thus Io, an electric conductor because of its ionosphere, interacts with both the Jovian magnetic field and the plasma torus.

[3] Auroral spots have been observed in the Jovian atmosphere at latitudes lower than the main auroral oval. The brightest of these spots maps roughly to the position of the footprint of the magnetic field line connecting Jupiter to Io and is thus related to the Io-Jupiter interaction. Other spots have been related to the magnetic footprint of Europa and Ganymede [Clarke et al., 2002].

[4] The Io-Jupiter interaction is the archetype of magnetosphere-satellite interactions and certainly the most con-

\footnotetext{
${ }^{1}$ Laboratory for Atmospheric and Space Physics, University of Colorado at Boulder, Boulder, Colorado, USA.

${ }^{2}$ LPAP, Institut d'Astrophysique et Géophysique, Université de Liège, Liège, Belgium.

${ }^{3}$ Geophysical Institute, University of Alaska Fairbanks, Fairbanks, Alaska, USA.

Copyright 2010 by the American Geophysical Union. 0148-0227/10/2009JA014928
}

strained example of auroral phenomenon. Observations made in wavelengths ranging from UV to low-frequency radio have been providing information about the auroral emissions occurring at both Jupiter and Io. The Io footprint was first observed in infrared (hereafter IR) by Connerney et al. [1993] with the NASA's IRTF using a narrowband filter, centered on one of the $\mathrm{H}_{3}^{+}$bands near $3.4 \mu \mathrm{m}$. The $\mathrm{H}_{3}^{+}$rotationalvibrational emission is the main auroral emission occurring in IR at Jupiter. It is generated by the collision of energetic, magnetospheric electrons with the ionosphere particles and by Joule heating. The observations do not resolve the Io footprint size, but they do reveal substructures (see section 2.1 and Figure 1).

[5] Only a few observations of the visible part of the Iocontrolled aurorae have been performed. This is due to the fact that visible observations of the aurorae have to be performed on the night side of the planet. However, Ingersoll et al. [1998] and Vasavada et al. [1999] presented observations of the visible Io spot by the Galileo spacecraft.

[6] In the UV, the Io footprints were discovered by Clarke et al. [1996] and Prangé et al. [1996] and are still observed with the Hubble Space Telescope (Figure 2). Since their discovery, many observations have been made so that the UV emissions are now the most well-documented. The emissions are due to magnetospheric electron precipitation on the top of the Jovian atmosphere. The electrons collide with the ionospheric hydrogen, exciting emissions in the hydrogen bands. The UV observations have a good angular resolution, even if the UV spot is not fully resolved. This (relatively) high resolution permits the observation of the footprint substructures (see section 2.1). The numerous observations of the UV spots have also led to several estimates of the radiated and precipitated powers associated with the Io spot. These will be discussed in section 2.2. 


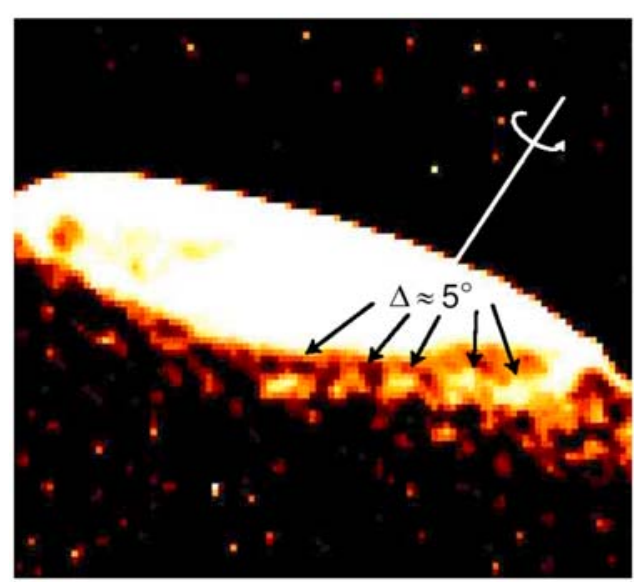

Figure 1. Observation of the IR aurorae of Jupiter; the bright spots result from the Io-Jupiter interaction. Secondary (RAW) spots appear to be spaced by $5^{\circ}$ in longitude. Adapted from Connerney and Satoh [2000].

[7] Radio emissions are not related to the footprint itself but to the adjacent magnetic field lines. The Jovian radio emissions were first detected by Burke and Franklin [1955], and their control by the Io magnetic longitude was established by Bigg [1964]. The decameter part of the emission ( 1 to $40 \mathrm{MHz}$ ) is the most intense and is related to Io. The radio frequencies correspond to the gyrofrequencies of electrons along the magnetic field lines and thus are thought to be due to the Cyclotron-Maser Instability (CMI), as in the terrestrial auroral emissions [Zarka, 1998; Treumann, 2006, and references therein]. The Io-controlled decameter emissions are composed of long-duration "arcs" and millisecond bursts (Figure 3). Both kinds of radio emissions can be used to determine the precipitated power and the precipitating electron energy (see section 2.2), as well as information about their location and the existence of substructures in the footprint (see section 2.1).

[8] Finally, flybys of Io by the Galileo spacecraft brought complementary information about the particle distribution in the dense plasma torus surrounding the orbit of Io [Williams et al., 1996, 1999; Williams and Thorne, 2003; Frank and Paterson, 1999; Mauk et al., 2001].

[9] The physics involved in the Io-Jupiter interaction is well-constrained, as both the sources (Io-magnetosphere interaction) and sinks (auroral precipitation) of power are known. The transmission of the power between Io and Jupiter is thus the main subject to be examined and is the purpose of the present paper.

[10] In the first section we will summarize the UV and radio observations as they relate to auroral morphology, radiated power, and the energy of the precipitating particles. In the following section we present the theoretical models that have been proposed to model the Io-Jupiter interaction: The description(s) of the local Io-plasma-magnetic field interaction (section 3 ), the description(s) of the power transmission from the Io to Jupiter (section 4), and finally the description(s) of the particle acceleration (section 5). From the model comparisons and discussions we introduce a model for the Io-Jupiter interaction that is global and consistent. This model involves the filamentation of the Alfvén waves packets generated by the Io-Jupiter interaction. In section 6 we compare the predictions of the model to the observations.

\section{Jovian, Io-Related Aurorae}

\subsection{Morphology of the Aurorae}

[11] Most of the observations are performed on the ground or in the Earth vicinity, in which case the angular resolution is too low to correctly resolve the Io footprint. Nevertheless, all observations from UV to IR show that the Io footprint consists of a main spot ("main Alfvén wing (MAW) spot"), secondary spots ("reflected Alfvén wing (RAW) spots"), and a diffuse tail that has an extent of some tens of degrees [Connerney and Satoh, 2000; Gérard et al., 2006]. Moreover the observations, in particular the UV, tend to show that the MAW spot has a typical radius of 100-200 km, elongated in the longitudinal direction, which matches (at least in the latitudinal direction) the size of the Io disk projected onto the ionosphere by following the magnetic field lines, i.e., angular size of $0.5^{\circ}-0.9^{\circ}$ on the planet [Gérard et al., 2006].

[12] The RAW spots have been observed in UV [Gérard et al., 2006] and IR [Connerney and Satoh, 2000] (Figure 1). When Io is near the center of the torus, IR observations seem to show almost equidistant spots separated by $5^{\circ}-6^{\circ}$ [Connerney and Satoh, 2000] (Figure 1). In the UV, the RAW spots are mainly observed when Io is on the border of the torus. In this case the first RAW spot is near the MAW spot, ultimately merging with it, whereas the second spot is shifted by $\sim 12^{\circ}$ in longitude. These spots are commonly interpreted as Alfvén wave reflections in the torus [Neubauer, 1980].

[13] A third class of spot has been observed in UV, which in the present paper will be called "transhemispheric electron beam spot" (TEB spot), following Bonfond et al. [2009]. It is sometimes called a precursor, but because it can trail the MAW spot, this name is inappropriate. A detailed description of the characteristics of this spot can be found in the work of Bonfond et al. [2008]. The brightest TEB spot has a longitudinal shift relative to the MAW spot that varies from about $-2.5^{\circ}$ to $+2.5^{\circ}$. The fact that this spot could precede the MAW spot implies that it cannot be due to Alfvén wave reflection. Other spots shifted by multiples of $\sim 5^{\circ}$ may exist but are not detected yet.

[14] The decameter long-duration emissions are due to energetic electrons emitting along an "active" magnetic field

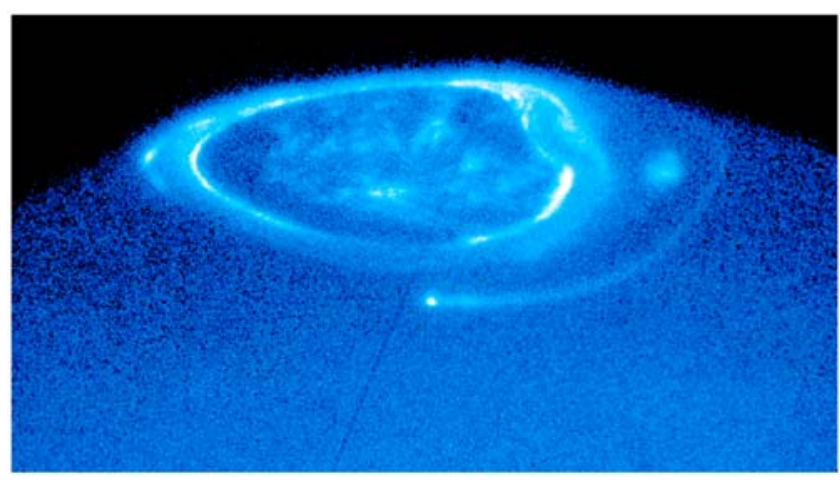

Figure 2. Observation of the UV aurorae of Jupiter. The bright spot with an elongated tail results from the Io-Jupiter interaction. 

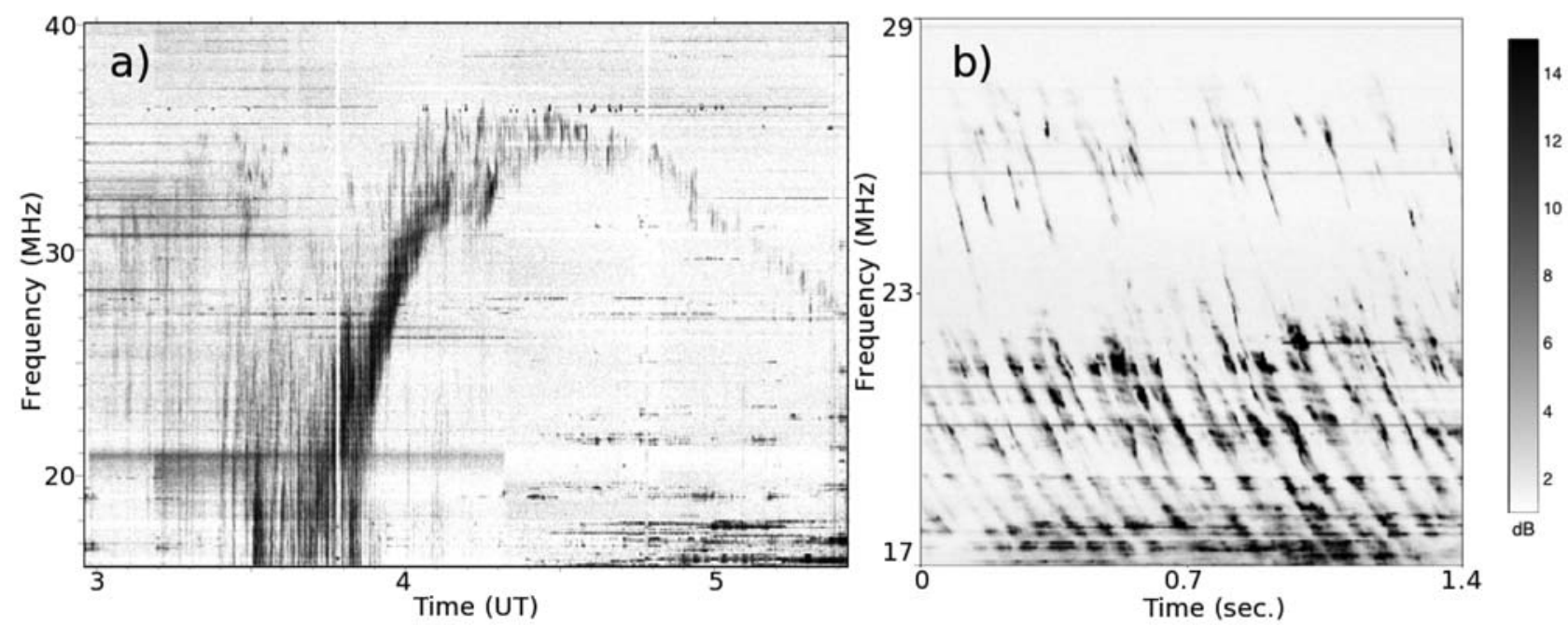

Figure 3. Observations of the radio emission of Jupiter related to Io. (a) Long-duration arc. (b) Millisecond bursts. Adapted from Hess [2008].

line [Lecacheux et al., 1998; Queinnec and Zarka, 1998; Hess et al., 2008]; they have an average lead angle of a few tens of degrees with respect to Io longitude [Queinnec and Zarka, 1998; Hess et al., 2008]. Owing to the localized emission and the strongly anisotropic pattern of the radio beaming, the Io-controlled radio emissions appear arc-shaped in the time-frequency plane. Several succesive arcs may appear during the observations. Their separation has been interpreted as due to several active field lines separated by $\sim 5^{\circ}$. This value has been confirmed by Hess et al. [2010], who have modeled the radio emission and compared it to the observations.

[15] Figure 4 shows a summary of the locations of the Jovian auroral spots in radio, IR, and $\mathrm{UV}$, and their relation to the trajectory of the Alfvén wave generating them (in green) as proposed by Gurnett and Goertz [1981]. Figure 4 also shows the locations of the acceleration regions and of the resulting electron beams, which are discussed in the present paper. In the following sections we will present only the observations about the brightness of the electron beams and the related powers of the MAW spot emissions.

\subsection{Auroral Power and Precipitating Particle Energy}

[16] A summary of the values of the radiated and precipitated powers found in the literature is shown by Table 1 .

\subsubsection{On the Spot: From UV to IR}

[17] The best determination of the power involved in the auroral emissions has been made in UV. The UV aurorae on Jupiter, as on others planets, are due to the precipitation of energetic particles from the magnetosphere into the planetary ionosphere. In the case of the Io-induced emissions, these particles are mainly electrons. The relation between power and brightness is difficult to obtain and depends on models of the collision of the precipitating electrons with the ionospheric particles. Thus the energy of the precipitating electrons is an important variable.

[18] For low-energy electrons the collisions occur at the top of the ionosphere, whereas for those with higher energies the collisions occur at lower altitudes. The emissions due to high-energy electrons have to travel a longer path length through the Jovian ionosphere, with the result that their spectra may present observable absorption lines, whereas emissions due to lower-energy electrons may not. The main absorption lines used to deduce the electron energy are the $\mathrm{CH}_{4}$ lines, which are easily observable. The intensity of the absorption by $\mathrm{CH}_{4}$ is usually measured by the color ratio of the spectra [Yung et al., 1982; Gérard et al., 2002, and references therein]. To relate the color ratio to the altitude of the emissions and then to the electron energies, a model of the atmosphere in the auroral region is needed. Such models are difficult to establish since there are very few observations to constrain them. In particular, concentration of minor species such as $\mathrm{CH}_{4}$ is uncertain. However, using realistic assumptions for the atmospheric model, relations between the color ratio of the UV emissions, altitude, and particle energy have been made [Dols et al., 2000; Gérard et al., 2002]. Note that the color ratio is almost the same in the spots and in the tail. Thus the altitude and the related electron energy of the emissions must be comparable for both regions.

[19] Color ratios have long been used to determine the electron energies involved in the Jovian aurorae [Clarke et al., 1996, 1998; Prangé et al., 1996, 1998; Dols et al., 2000; Gérard et al., 2002]. A mean value of a few tens of $\mathrm{keV}$ has been found for the precipitating electron energy. However, Bonfond et al. [2009] have proposed a more direct method to measure the altitude of the emissions. This method requires observations of Io's tail when it passes across the limb plane, providing a measurement of the maximum altitude of the emissions relative to the disk edge. Results of this method are not dependent on models and assumptions. These direct measurements have shown that the tail emission occurs $\sim 900 \mathrm{~km}$ above the 1 bar level, almost four times higher than predicted by the atmospheric models. Bonfond et al. [2009] showed that reaching this altitude requires the mean electron energy to be around $1 \mathrm{keV}$, in a Kappa $(\mathrm{K}=2.3)$ distribution, and at a temperature of $100 \mathrm{eV}$. Since the color ratio is almost the same for the spots and the tail, we infer that these results apply to the MAW spot too. 


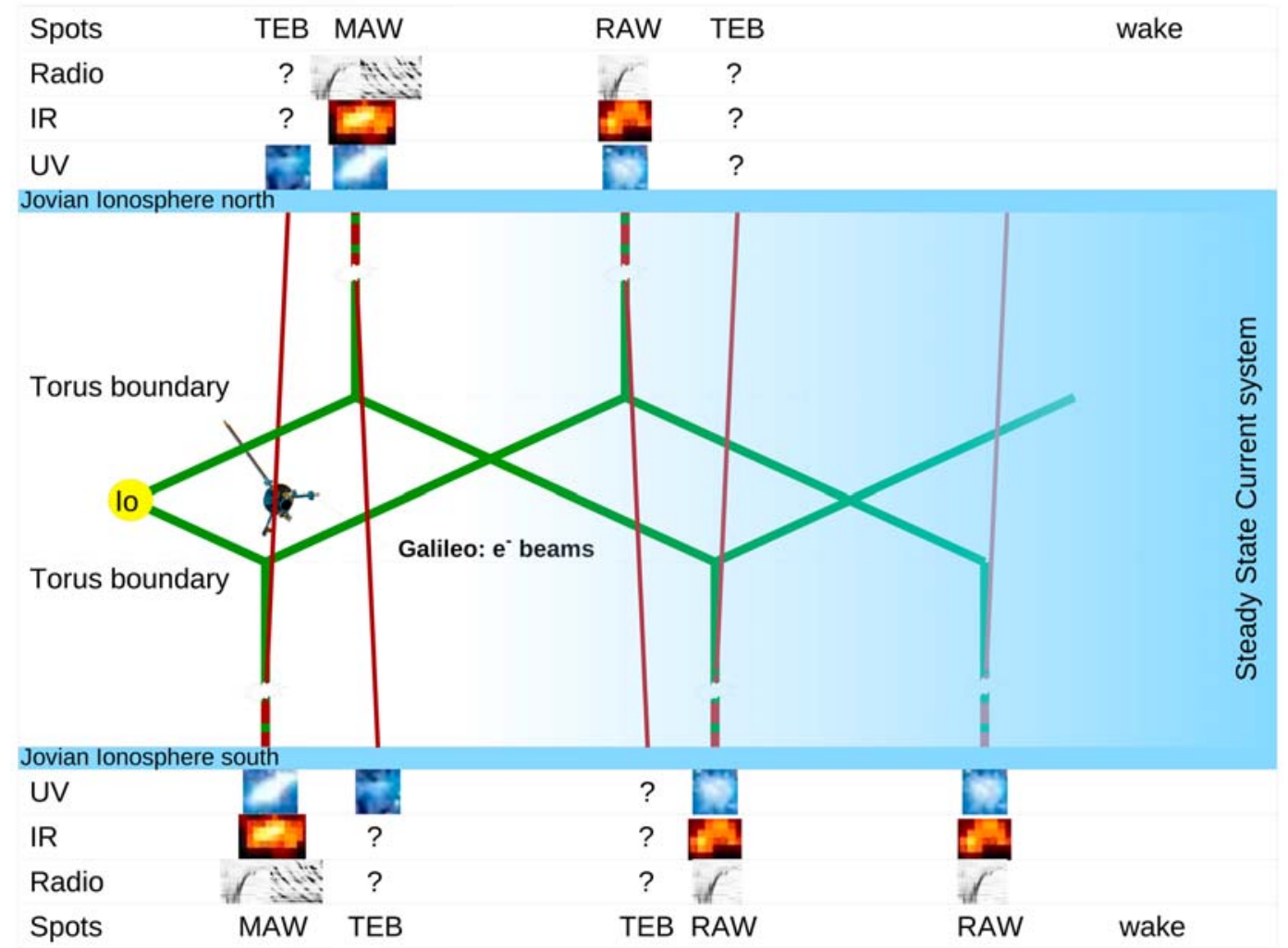

Figure 4. Sketch summarizing our interpretation of the Io-Jupiter interaction in the filamentary Alvén wave model. Green lines indicate the currents carried by Alfvén waves, whereas the red to purplish lines stand for the currents carried by particles. The associated emissions in UV, IR, and radio in the Jovian northern and southern hemispheres are shown on the top and on the bottom of the sketch, respectively. Question marks indicate emissions not observed or not confirmed or (for the northern radio arcs) location of emission unknown. In addition, the location of Galileo during the energetic electron beams observation in Io's wake is indicated by the spacecraft picture.

[20] From the UV observations, the required power of the electron precipitation varies from $\sim 33 \mathrm{~mW} / \mathrm{m}^{2}$ in the MAW spot to $\sim 6 \mathrm{~mW} / \mathrm{m}^{2}$ in the tail [Gérard et al., 2002], while the mean electron energy remains almost constant. When integrating this value on the MAW spot (approximated by a circle with a radius of $100-200 \mathrm{~km}$ ), we obtain a mandatory energy flux of the precipitating electrons of a few $10^{9} \mathrm{~W}$. Taking into account experimental uncertainties, the total power of the MAW spot emission can estimated to be from $\sim 10^{8}$ to a few $10^{9} \mathrm{~W}$. Most observations are consistent with a total emitted flux on the order of or larger than $10^{9} \mathrm{~W}$. In former studies, the low angular resolution of the UV observations indicated that Io's MAW spot could have a $1000 \mathrm{~km}$ radius, leading to values of $\sim 10^{10} \mathrm{~W}$ emitted and $\sim 10^{11} \mathrm{~W}$ precipitated [Clarke et al., 1996]. However, when integrating over all spots and the tail, the total precipitated power is $\sim 10^{11} \mathrm{~W}$ in the UV. This integrated value is consistent with the IR estimations [Satoh and Connerney, 1999].

[21] The power of the visible emissions has been observed to be $\sim 10^{8} \mathrm{~W}$ [Ingersoll et al., 1998; Vasavada et al., 1999]. Although no estimation by IR of the power of the MAW spot alone has been made, $t \mathrm{t}$ is thought to be of the same order as UV estimates.

\subsubsection{Observations of the Radio Emissions}

[22] The long-duration radio arc shape is thought to be a consequence of the effects of the strongly anisotropic beam-

Table 1. Summary of the Powers Emitted in Different Wavelength Ranges and the Corresponding Precipitated Power for the Main Spot Only and for the Whole Io-Controlled Emissions

\begin{tabular}{lrrrr}
\hline Wavelength Range & Main Spot Emitted Power & Main Spot Precipitated Power & Spots + Tail Precipitated Power & Electron Motion \\
\hline UV & $\sim 10^{9} \mathrm{~W}$ & few $10^{9}-$ few $10^{10} \mathrm{~W}$ & $\sim 10^{11} \mathrm{~W}$ & planetward \\
IR & $10^{8} \mathrm{~W}$ & $?^{\mathrm{a}}$ & $\sim 10^{11} \mathrm{~W}$ & $?$ \\
Visible & $10^{9}-10^{10} \mathrm{~W}$ & $\sim$ & $?$ & planetward \\
planetward \\
Radio & not relevant & $\sim 10^{10} \mathrm{~W}$ & $10^{10}-10^{11} \mathrm{~W}$ & $?$ \\
Beams & $\sim 10^{10} \mathrm{~W}$ & $\sim 10^{10} \mathrm{~W}$ & antiplanetward \\
Total & few $10^{10} \mathrm{~W}$ & few $10^{11} \mathrm{~W}$ & \\
\hline
\end{tabular}

${ }^{\mathrm{a}} \mathrm{IR}$ powers for the main spot are generally thought to be comparable to the UV ones. 
ing pattern of the radio emissions. The CMI theory states that the emission pattern is a hollow cone with width $\sim 1^{\circ}$. The geometry of the observation plays an important role; in particular, the lead angle between the instantaneous Io magnetic field line and the emitting field line strongly affects the arc shape. Moreover, Hess et al. [2008] have shown that the arc shape can be well reproduced by assuming an emission for which the beaming pattern varies with frequency and is dependent on the particle velocity. The energy of the emitting electrons has been deduced to be a few $\mathrm{keV}$ from the timefrequency shape of the decameter emissions [Hess et al., 2010].

[23] On the other hand, the drift in frequency of the short bursts can be related to the motion of the emitting electrons along the magnetic field lines (since the emission frequency is related to the magnetic field intensity). Measuring the drift rate thus permits a measurement of the velocity of the electrons parallel to the magnetic field lines. The variation with frequency shows that the electrons are in adiabatic motion, except in localized ranges in which electric potential structures may exist [Hess et al., 2007a]. From the parallel velocity profile versus frequency it is possible to deduce that the total energy of the particles is 2-5 keV [Zarka et al., 1996; Hess et al., 2007a, 2009b]. This value is consistent with the fit of the long-duration arcs and with the value obtained from UV observations.

[24] The power emitted in radio has been found to vary from $3 \times 10^{9} \mathrm{~W}$ to $3 \times 10^{10} \mathrm{~W}$ [Queinnec and Zarka, 2001]. The energy flux of the precipitating particles needed to obtain these values is hard to determine because the efficiency of the CMI (at the origin of the emission) depends on the electron distribution. A good estimate of this efficiency varies from a few percent (for a loss cone distribution) to nearly $50 \%$ (for a ring or a shell distribution) [Pritchett et al., 2002]. From simulations and observations, Hess et al. [2007a, 2007b] deduced that the radio emission should be due to quasi-monoenergetic electrons, leading to a ring-like distribution with an efficiency of nearly $50 \%$ and requiring an energy flux of the precipitating electrons of few $10^{10} \mathrm{~W}$.

\subsubsection{Particle Measurements}

[25] Bidirectional electron beams aligned with the magnetic field were discovered in the wake of Io during the J0 Galileo flyby in 1995. Williams et al. [1996] reported observations of these beams between 15 and $150 \mathrm{keV}$, using EPD on board Galileo. Frank and Paterson [1999], using the PLS instrument on board the spacecraft, reported the detection on the same flyby of lower-energy electron beams between $100 \mathrm{eV}$ and $10 \mathrm{keV}$. Mauk et al. [2001] combined the lower-energy and high-energy beams measurements to provide a full distribution function and particle flux. Both lower-energy electron beams were also detected above Io's poles and along its flanks during the Galileo flybys of the satellite (B. Paterson, private communication, 2009) [Williams and Thorne, 2003]. As the low-energy beams were detected in the wake, over the poles, and along the flank of Io, the spatial distribution can be estimated to cover roughly the whole cross section of Io $\sim 10^{13} \mathrm{~m}^{2}$, although as the wake observations suggest, the beams location is probably slightly downstream of Io and slightly larger.

[26] Frank and Paterson [1999] reported lower-energy electron beams (between $100 \mathrm{eV}$ and $10 \mathrm{keV}$ ) parallel to the magnetic field lines and displaying a smooth peak at $\sim 350 \mathrm{eV}$. The published energy flux integrated on a $20^{\circ}$ half-opening cone is $\sim 2 \mathrm{~mW} / \mathrm{m}^{2} \mathrm{sr}^{-1}$ in both directions. If we follow Williams et al. [1999] and assume that beams are confined in a $6^{\circ}$ opening cone $(0.034 \mathrm{sr})$, then the specific energy flux $\sim 60 \mathrm{~mW} / \mathrm{m}^{2} \mathrm{sr}^{-1}$.

[27] We compute the total precipitated power at Jupiter by taking into account only the low-energy electron fluxes, which are much larger than the high-energy electron fluxes. The energy flux at Io, projected onto Jupiter with a mirror ratio equal to 400 would be $800 \mathrm{~mW} / \mathrm{m}^{2}$ and the total power precipitated on a circular spot with a radius of $100 \mathrm{~km}$ would be $2.4 \times 10^{10} \mathrm{~W}$.

[28] These upper limits of the electron energy fluxes at Jupiter and of the total power in the field-aligned beams are comfortably above the power deduced from the UV auroral emissions in the MAW spot (a few tens of $\mathrm{mW} / \mathrm{m}^{2}$ of precipitated electrons and a total power of $\sim 10^{9} \mathrm{~W}$ ).

[29] An estimation of the portion of the beams in a 3 loss cone, based on a 6 half-width of a gaussian distribution in pitch angle [Williams et al., 1999, Figure 8], would reduce the energy flux of the precipitated electrons to $30 \%$ of their maximum value, still well above the constraints deduced from UV observation.

[30] Potentially, some part of the beams observed at Io might be the transhemispheric beams generating the TEB spots. Bonfond et al. [2008] addressed the energy comparison of the beams and the leading spot emission. Their conclusion is that the power of the beams is sufficient for generating TEB spot.

\section{Local Interaction}

\subsection{Electric Field Generation Across Io}

[31] Special relativity states that an electric field $(E)$ exists in a reference frame in which the background magnetic field $(B)$ moves with a velocity $\delta_{v}$ :

$$
\mathbf{E}=-\delta \mathbf{v} \times \mathbf{B}
$$

The effect of this field on charged particles initially at rest in this reference frame results in a drift of the particles at the velocity of the magnetic field.

[32] This effect is relevant for describing a plasma that can freely follow the magnetic field line motion, such as the Io plasma torus far from the satellite. With respect to Io, the corotating plasma has a velocity of $v_{I o}=57 \mathrm{~km}$.

[33] In the ideal case of a laminar, almost unperturbed, flow of plasma, the electric field $\left(E_{I o}\right)$ is localized across the Io disk and is determined from equation (1) with $\delta_{v}=v_{I o}$. Assuming an average value for $B$ of $2 \mu \mathrm{T}$, the electric field across Io is approximately $0.01 \mathrm{~V} / \mathrm{m}$.

[34] However, this is a simplified model of the Io-Jupiter interaction. A more complete description of the flow of plasma around Io and its atmosphere must include mass loading due to charge exchange and ionization. An electric field is generated in the perturbed plasma until the ionospheric plasma is accelerated to corotation (equation (1)) [Saur et al., 1999, 2002a; Saur, 2004; Delamere et al., 2003; Dols et al., 2008]. 


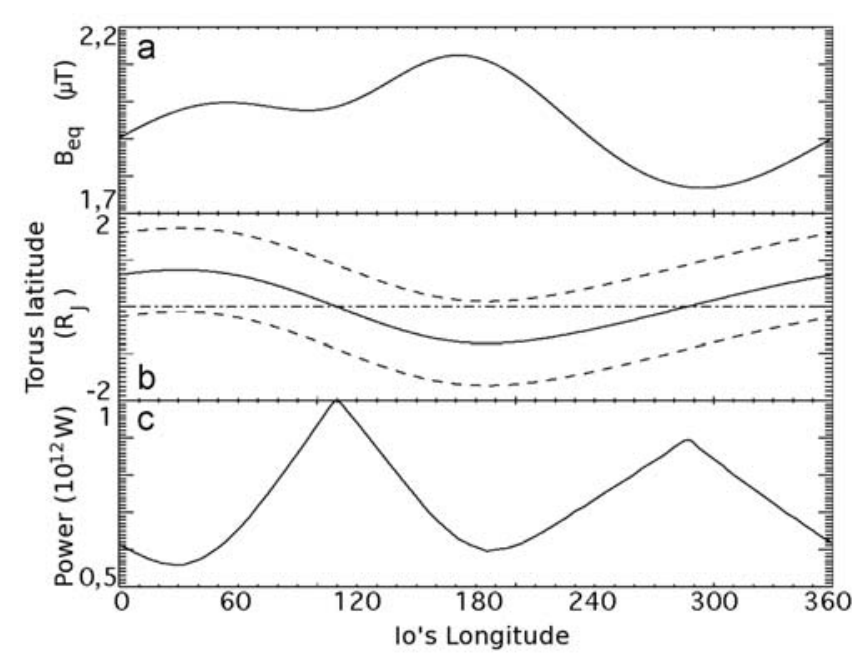

Figure 5. (a) Magnetic field at Io versus Io's longitude (VIP4 magnetic model [Connerney et al., 1998]). (b) Position of the centrifugal equator relative to Io's orbit versus Io's longitude (solid lines). The dashed lines stand for one torus height scale $\left(1 R_{J}\right)$. (c) Power of the Io-Jupiter interaction versus Io's longitude. Adapted from Hess [2008].

\subsection{Current Generation}

[35] The electric field induces a current $(J)$ through Io. The induced current creates a perturbation of the magnetic field, resulting in a deformation of the magnetic field lines. This perturbation of the magnetic field lines can be modeled by an Alfvén wave packet, which will carry the current generated at Io. The Alfvén conductance is given by:

$$
\Sigma_{A}=\sqrt{\frac{\rho}{\mu_{0} B^{2}}}
$$

where $\rho$ is the plasma density. The integrated current carried by the Alfvén waves can be obtained from the Ohm voltagecurrent relation [Neubauer, 1980; Goertz, 1983; Saur, 2004]. For each hemisphere the current is given by:

$$
J=2 E_{I o} R_{I o} \Sigma_{A} \sim 1 M A
$$

This value of the electrical current is consistent with those deduced from the measurements of Voyager 1 [Acuna et al., 1981]. This relation neglects the finite conductance of the rest of the current circuit, even if the Alfvén wave-driven current is thought to close partly along the field lines and partly in the Jovian ionosphere. A more accurate description has been proposed by Saur [2004] to describe the current circuit.

[36] Such a relation involves the conductance integrated along the whole circuit and is mostly relevant in the case of a steady state current system. Because the information concerning the conductance value in the Jovian ionosphere, for example, takes more than several minutes to reach the Io orbit, this model may be more appropriate to describe the current circuit in the Io's wake. Numerical computations and magnetic perturbation measurements [Kivelson et al., 1996; Gurnett et al., 1996] show that the integrated conductance is mostly limited by the low conductance in the neighborhood of Io; thus, computing the current by using equation (3) or the Saur [2004] model gives similar results.

\subsection{Power Generated at Io}

[37] Since we know both the voltage across Io and the current it generates, we are able to compute the power radiated as Alfvén waves by the Io-Jupiter interaction:

$$
P=4 E_{I o}^{2} R_{I o}^{2} \Sigma_{A} \sim 10^{12} W
$$

The computation of the power gives values of about $10^{12} \mathrm{~W}$, which depend on magnetic field intensity (Figure 5a) and the plasma density (which varies with the distance of Io relative to the torus center) (Figure 5b). The longitudinal dependence of the power is shown in Figure $5 \mathrm{c}$. The power varies by a factor of two between its maxima and its minima. The maxima correspond to the longitudes at which Io is in the center of the torus (high-density $\rho$ ), whereas its minima occur when Io is on the borders of the torus (low density).

\section{Power Transmission}

\subsection{Alfvén Wave Propagation}

[38] The Alfvén waves propagate at the Alfvén velocity, which in ideal MHD is both the group and phase speed:

$$
v_{a}=\frac{B}{\sqrt{\mu_{0} \rho}}
$$

When Io is in the middle of the torus, i.e., for $\rho \simeq$ $2000 \mathrm{~m}_{i} \mathrm{~cm}^{-3}$, where $m_{i}$ the ion mass [Bagenal, 1994; Moncuquet et al., 2002], the Alfvén velocity is $\sim 200 \mathrm{~km} / \mathrm{s}$. As the wave propagates out of the torus, the density $\rho$ decreases and the magnetic field $B$ increases. Thus the wave velocity increases. At the edge of the torus the density is low enough for the Alfvén velocity to reach the speed of light, and a relativistic expression of the Alfvén velocity must be used:

$$
v_{a}=\frac{B}{\sqrt{\mu_{0} \rho+\frac{B^{2}}{c^{2}}}}
$$

[39] The computation of the Alfvén velocity through the torus shows that it takes several minutes for the wave to escape the torus, and the trajectory of the Alfvénic perturbation in Io's frame appears oblique relative to the background magnetic field direction, generating a pattern called Alfvén wings. Out of the torus, the Alfvén waves propagate along the magnetic field lines at nearly the speed of light, carrying the current generated at Io to the Jovian ionosphere. The Alfvén wave phase velocity depends on kinetic and inertial terms:

$$
v_{\phi, a}^{2}=v_{a}^{2} \frac{\left(1+k_{\perp}^{2} \rho_{s}^{2}\right)}{\left(1+k_{\perp}^{2} \lambda_{e}^{2}\right)}
$$


where $k_{\perp}$ is the perpendicular component of the wave vector $k, \rho_{s}$ is the ion acoustic gyroradius (kinetic term), and $\lambda_{e}\left(=c / \omega_{p e}\right)$ is the electron inertial length (inertial term).

[40] As the wave phase velocity changes, the refractive index of the plasma changes too. This implies partial reflection of the Alfvén waves. The two main index gradients are located at the edges of the torus and at the Jovian ionospheric boundaries. The reflection coefficient depends on the index variation, i.e., on the wave phase speed variation. Numerical simulations give a power transmission coefficient, for wavelengths on the order of Io's diameter, of $20-25 \%$ [Wright, 1987; Delamere et al., 2003] from Io to the Jovian ionospheric boundary. As the reflection coefficient on the torus boundary is high, the reflected Alfvén wave model, which explains the presence of multiple spots, seems valid if we set apart the TEBs. However, such a high reflection is an issue regarding the power constraint, since $\sim 80 \%$ of the wave power is trapped in the torus. We showed in the previous section that several percent of the wave power must be transferred to the particles. Thus, for wavelengths of Io's scale, the particles must be accelerated in the torus, or the acceleration process must have several tens of percents of efficiency. Another solution is to consider wavelengths that are less reflected.

\subsection{Transmission Coefficients}

\subsubsection{Dependence on $\boldsymbol{k}_{\|}$}

[41] The reflection coefficient (in amplitude) of a wave propagating in an index gradient is obtained from the necessary continuity of both the electric and magnetic fields along the wave trajectory and is given by

$$
R_{a}=\frac{d \ln n}{2 d x} d x
$$

The transmission coefficient is obtained through conservation of energy. Thus the reflection and transmission coefficient in energy must be computed. The reflection coefficient in energy is obtained by squaring $R_{a}$. However, one must be careful about the ratio between the wavelength $\lambda_{\|}$and the scale of the index gradient $L$. For discontinuities $\left(L \ll \lambda_{\|}\right)$ the reflection coefficient in energy is

$$
R_{\epsilon ; D}=\left(\frac{n_{1}-n_{2}}{n_{1}+n_{2}}\right)^{2}
$$

In the case of the Io flux tube, the reflection coefficient between the center and the edge of the torus would then be $\sim 1$, whereas for smooth gradients $\left(L \gg \lambda_{\|}\right)$, the WKB approximation gives

$$
R_{\epsilon ; W K B}=\int_{0}^{L}\left(\frac{d \ln n}{2 d x} \delta x\right)^{2} \frac{d x}{\delta x} \sim 0
$$

with $\delta x \rightarrow 0$. As we see, the two asymptotic expressions of the reflection coefficient give opposite results. In the Io flux tube case, the parallel wavelengths corresponding to the Io size do not match any of these approximations. Thus we need to find an expression of the reflection coefficient that works for all $\lambda_{\|} / L$ ratios.
[42] The wavelength acts on the energy reflection coefficient because the energy of a wave is not defined on a scale smaller than its wavelength. Therefore the energy reflection coefficient must be computed from the amplitude reflected over one wavelength:

$$
R_{\epsilon ; \lambda}=\left(\int_{-\frac{\lambda_{\|}}{2}}^{\frac{\lambda_{\|}}{2}} \frac{d \ln n}{2 d x} d x\right)^{2}
$$

In the $L \rightarrow 0$ approximation (discontinuity), the gradient $\left(\frac{d \ln n}{2 d x}\right)$ tends toward a Dirac distribution and we retrieve the $R_{\epsilon ; D}$ expression. In the $\lambda_{\|} \rightarrow 0$ approximation (WKB), $R_{\epsilon ; \lambda_{\|} \rightarrow 0} \rightarrow\left(\frac{d \ln n}{2 d x}\right)^{2}$ and we retrieve the WKB expression of the energy reflection coefficient.

[43] This energy reflection coefficient is computed on a discontinuous space (since it is defined on a wavelength). To get a continuous expression of the energy reflection coefficient, we define

$$
R_{\epsilon}=\frac{1}{\lambda_{\|}}\left(\int_{-\frac{\lambda_{\|}}{2}}^{\frac{\lambda_{\|}}{2}} \frac{d \ln n}{2 d x} d x\right)^{2}
$$

If we assume a constant $\left(\frac{d \ln n}{2 d x}\right)$ gradient in the transition region (TR) of length $L$, this expression can be written as

$$
\begin{gathered}
\int_{(T R)} R_{\epsilon}=\frac{\lambda_{\|}}{L}\left(\frac{n_{1}-n_{2}}{n_{1}+n_{2}}\right)^{2} ; \lambda_{\|}<L \\
\int_{(T R)} R_{\epsilon}=\left(\frac{n_{1}-n_{2}}{n_{1}+n_{2}}\right)^{2} ; \lambda_{\|}>L
\end{gathered}
$$

This matches the MHD simulations of Wright [1987], which show that the reflection coefficient is high (almost 1) and constant for wavelengths larger than the torus height scale but decreases toward 0 for smaller wavelengths.

\subsubsection{Dependence on $\boldsymbol{k}_{\perp}$}

[44] In the previous section we computed the dependence of the transmission on $k_{\|}$and on the index gradient, but we did not express the index gradient itself. For an Alfvén wave, the index variable is $n=v_{\phi, a} / c$; the amplitude reflection coefficient is then

$$
R=\frac{d \ln v_{\phi, a}}{d x} d x
$$

where the phase velocity is given by equation (7). In the nonrelativistic approximation the phase velocity is given by

$$
v_{\phi, a}^{2}=\frac{B^{2}}{\mu_{0} m_{i} n} \frac{\left(1+k_{\perp}^{2}\left(\frac{m_{i} c_{s}}{e B}\right)^{2}\right)}{\left(1+k_{\perp}^{2}\left(\frac{c^{2} m_{e} \epsilon_{0}}{e^{2} n}\right)\right)}
$$

where $n$ is the plasma density, $m_{i}$ is the ion mass, $m_{e}$ is the electron mass, and $c_{s}$ is the speed of sound in plasma. As the 
wave travels out of the torus, the Alfvén velocity term $\left(v_{a}^{2}=\frac{B^{2}}{\mu_{0}, m_{i} n}\right)$ increases abruptly, as the magnetic field $B$ increases and the density $n$ decreases. In the meantime, the kinetic term $\frac{m_{i} c_{s}}{e B}$ decreases, whereas the inertial term $\frac{c m_{e} \epsilon_{0}}{n e^{2}}$ increases. Thus, the kinetic and inertial terms diminish the increase of phase velocity and its reflection. This effect is controlled by the value of the perpendicular wave number $k_{\perp}$. In the MHD limit $\left(\lambda_{\perp} \gg \rho_{s}, \lambda_{e}\right)$ this correction is null. In the small scale limit $\left(\lambda_{\perp} \ll \rho_{s}, \lambda_{e}\right)$ the phase velocity tends asymptotically toward

$$
v_{\phi, a}^{2} \rightarrow \frac{m_{i}}{m_{e}} c_{s}^{2}=\text { const }
$$

However, when the group velocity reaches the velocity of light, the relativistic correction leads to a phase velocity that varies along the magnetic field lines. From the expression of the phase velocity one can deduce that the transmission coefficient is almost 1 at the small scales $\left(\lambda_{\perp}<\rho_{s}, \lambda_{e}\right)$ can be low at large scales $\left(\lambda_{\perp}>\rho_{s}, \lambda_{e}\right)$. Figure 7 shows the transmission coefficient of the energy as a function of distance from the torus center and of different wavelengths. Shorter wavelengths are less reflected, thus allowing high-latitude acceleration.

\subsection{Inertial Alfvén Waves: Observations and Origins}

[45] Magnetic field perturbations, appearing as Alfvén waves, with scales much shorter than the Io's radius have been observed in the Io's plasma torus by the Galileo probe [Chust et al., 2005]. Such filamented waves have been observed by numerous spacecraft in various locations in the terrestrial magnetosphere [Louarn et al., 1994; Stasiewicz et al., 1997; Alexandrova et al., 2004; Chaston et al., 2005]. As pointed out by Wright [1987], these smaller scales may be able to escape the torus.

[46] The filamentary Alfvén waves are thought to be generated by turbulent filamentation of long-scale Alfvén waves over density gradients or density perturbations [Champeaux et al., 1997, 1998; Shukla and Stenflo, 2000; Shukla and Sharma, 2002; Sharma et al., 2008a]. This may happen on the border of the torus, where the density gradient is maximum. Moreover, Io generates a density perturbation by itself in the form of slow MHD modes, which have been observed in simulations of the Io-Jupiter interaction by Jacobsen et al. [2007]. They may participate in the generation of smaller-scale Alfvén waves.

[47] As pointed out by many authors [Sharma et al., 2008a, and references therein], the result of filamentation of Alfvén waves on a density gradient (i.e., on the torus border) is a power law distribution of the Alfvén wavelengths. The spectral index is found to be $-\frac{5}{3}$ by numerical simulations [Sharma et al., 2008a]. However, in a later paper Sharma et al. [2008b] showed that these values can be slightly modified when nonlinear processes such as Joule heating are taken into account. Moreover the authors showed that for low- $\beta$ plasma the spectral indexes are not the same when parallel or perpendicular to the background magnetic field. This has been theorized by Galtier et al. [2000] and Galtier [2009] and observed by Saur et al. [2002b]. The spectral indices in the Io torus case should then be $\sim-2$ perpendicular to the magnetic field and $\sim-\frac{5}{3}$ parallel to the magnetic field. In the following we will approximate the
Alfvén wave power spectra by using a Kolmogorov spectrum $\left(k^{-\frac{5}{3}}\right)$, both parallel and perpendicular to the magnetic field; and a $k^{-2}$ spectrum, both parallel and perpendicular to the magnetic field, the actual distribution at Io falling between these two modeled distributions. Modeling of filamentation in the Io torus is beyond the scope of this paper.

\subsection{Power Transmission Along the Flux Tube}

[48] For long-scale Alfvén waves the wavelength is large and the refraction is high, but because the filamented waves have shorter wavelengths, their reflection coefficient is lower. To determine the importance of wavelength on the physics of the Io-Jupiter interaction, we compute the transmitted power as a function of the wavelength of the Alfvén waves. As the exact distribution is unknown, we will make the computations for three distributions:

[49] 1. The first distribution is the unfilamented waves (long-scale Alfvén waves). At the time of the interaction with Io the Alfvén wave is a wave packet whose wave number peaks at $k_{0}=\pi / R_{I o}$. Assuming a Gaussian-shaped wave packet, of width approximately $2 R_{I o}$, the distribution is given by:

$$
f_{0}(k) \propto \exp ^{-\left(k-k_{0}\right)^{2} /\left(2 k_{0}^{2}\right)}
$$

[50] 2. The second distribution is the filamented waves obtained through a turbulent cascade, which presents a power law spectrum between the energy injection scale $k_{0}=\pi / R_{I o}$ and the dissipation scale $k_{i}=\omega_{p i} / c$ :

$$
\begin{aligned}
& f_{1}(k) \propto \exp ^{-\left(k-k_{0}\right)^{2} /\left(2 k_{0}^{2}\right)} k<k_{0} \\
& f_{1}(k) \propto k^{-\alpha} k_{0}>k>k_{1} ; \alpha=2, \frac{5}{3} \\
& f_{1}(k) \propto \exp ^{-\left(k-k_{i}\right)^{2} /\left(2 k_{0}^{2}\right)} k>k_{i}
\end{aligned}
$$

[51] 3. The third distribution is the secondary short-scale distribution due to the filamentation of the long-scale Alfvén wave by the compressional modes. These modes have a typical wave number $k_{i}=\omega_{p i} / c$, and the distribution can thus be written

$$
f_{2}(k) \propto \exp ^{-\left(k-k_{i}\right)^{2} /\left(2 k_{0}^{2}\right)}
$$

A realistic distribution can be approximated, including some energy dissipation $\epsilon$, as follows:

$$
\begin{gathered}
f(k)=\alpha_{0} f_{0}(k)+\alpha_{1} f_{1}(k)+\alpha_{2} f_{2}(k) \\
1-\epsilon=\alpha_{0}+\alpha_{1}+\alpha_{2}
\end{gathered}
$$

[52] The transmission coefficient for each of these distributions is calculated using the equations developed in section 4.2 , the density profile shown in Figure 6 , and a magnetic field given by the VIP4 model [Connerney et al., 1998] for a magnetic field line longitude of 180 at the equator. Figure 7 shows the power transmission as a function of the wavelength for different distances (along the magnetic 


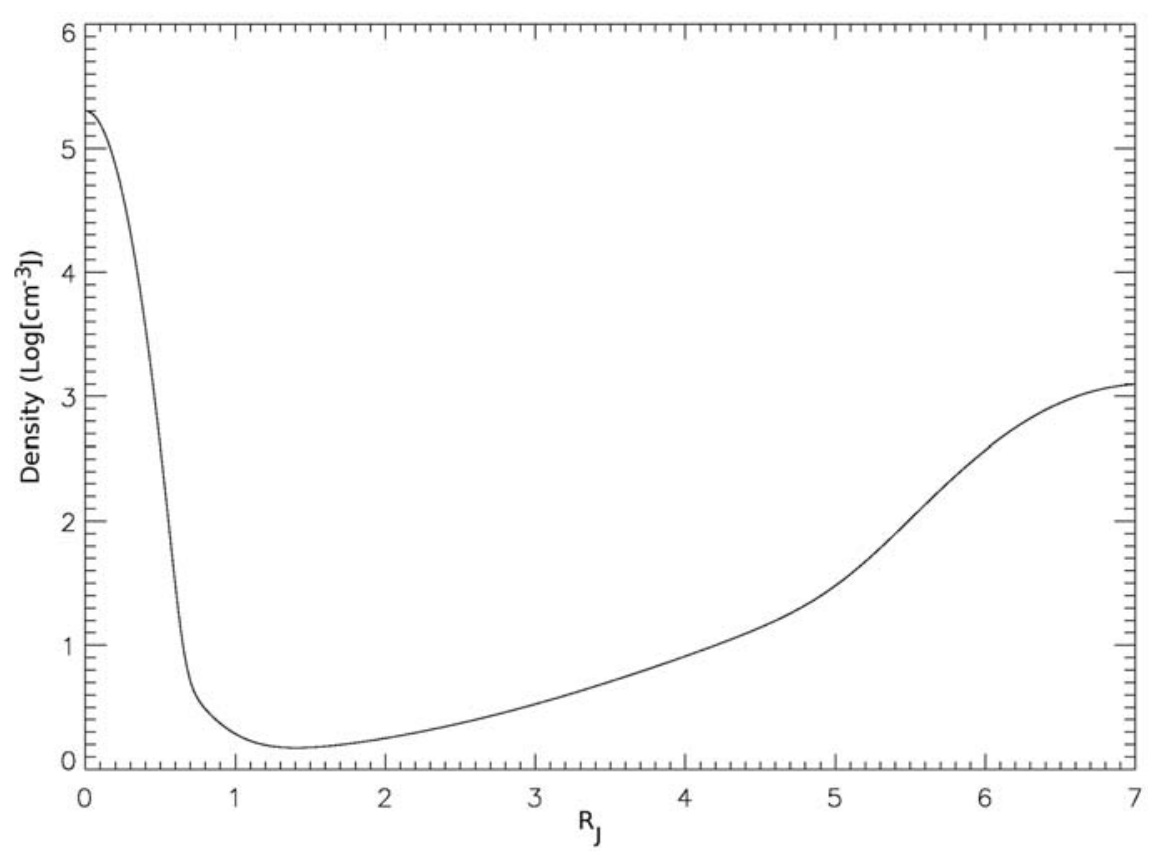

Figure 6. Density profile along the magnetic field line used in the present study. The distance along the field lines is defined from the top of the Jovian ionosphere. Io orbits at $7 R_{J}$. Approximated from torus models [Bagenal, 1994; Moncuquet et al., 2002] and simulations [Su et al., 2003].

field lines) from the torus. The waves with parallel wavelengths on the order of or larger than the Io radius are almost completely reflected. The perpendicular wavelength plays a less important role, even though smaller wavelengths are slightly less reflected than larger wavelengths.

[53] We are interested in the results at $6 R_{J}$ from the torus center ( $1 R_{J}$ above the Jovian ionosphere). We computed for each of the three previously defined distributions the total power reaching this altitude: for the long-scale distribution $\left(f_{1}\right)$ the power transmitted to the acceleration region is $\sim 17 \%$ (consistent with the previous estimations [Wright, 1987]), for the filamented distribution $\left(f_{2}\right)$ the transmission is between $\sim 45 \%$ (when $\alpha=2$ ) and $\sim 50 \%$ (when $\alpha=5 / 3$ ) and for the short-scale distribution $\left(f_{3}\right)$ the torus is almost transparent, $\sim 90 \%$ of the power reaching the acceleration region. We see from our computations that significant transmission
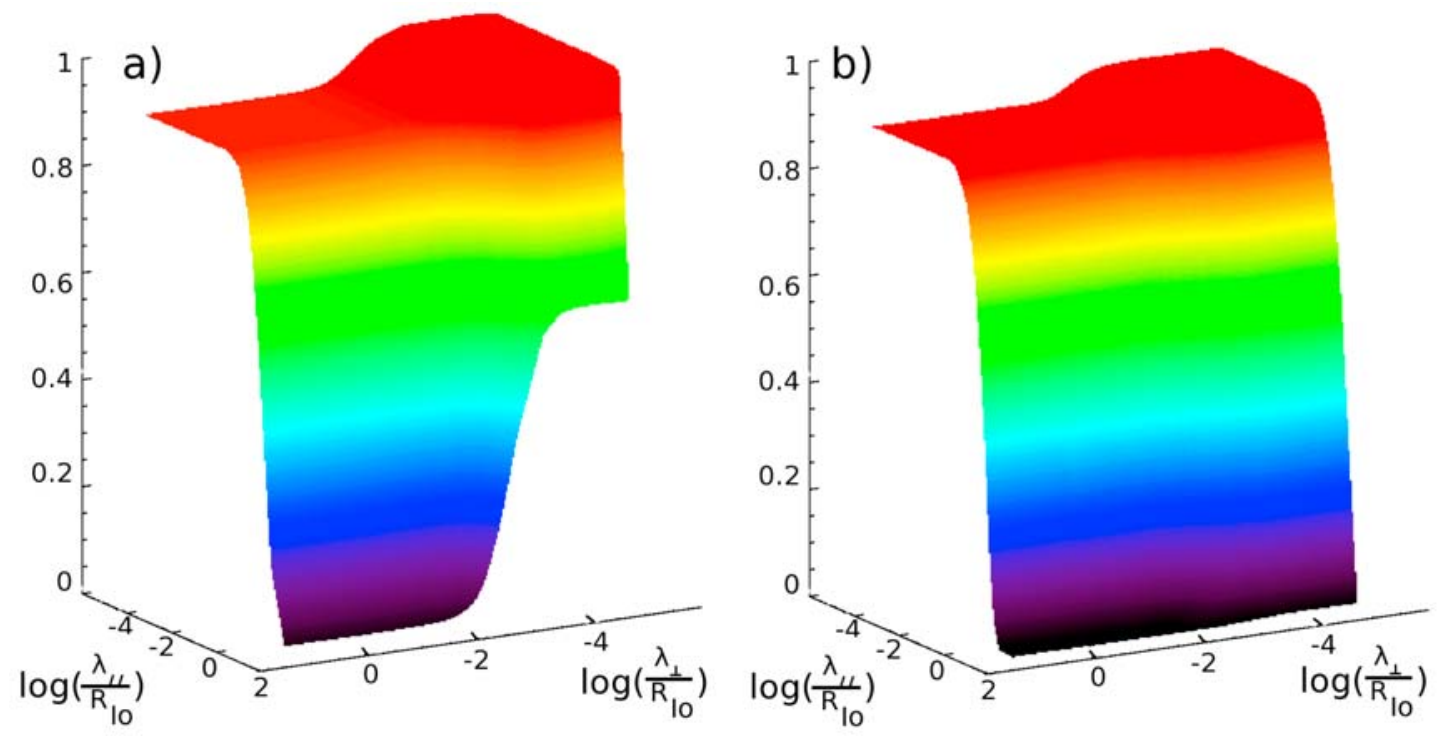

Figure 7. Power carried by the Alfvén waves transmitted at two distances $(s)$ from the torus center versus the parallel and perpendicular wavelengths: (a) $s=2 R_{J}$, just outside the torus; (b) $s=6 R_{J}$, near the acceleration region. The wavelengths vary from $20 R_{I o}$ to $10^{-5} R_{I o}$. The long $\left(\gtrsim R_{I o}\right)$ parallel wavelengths are almost completely reflected. The vertical axes correspond to the (dimensionless) transmission coefficient. 
is possible for filamented waves, although a nonnegligible part of the waves is reflected.

\section{Particle Acceleration}

\subsection{Particle Acceleration by Long-Scale Alfvén Wave in the Torus}

[54] The parallel electric field emerges from the difference between the wave group velocity and the phase velocity (equation (7)). The complete expression of the parallel electric field associated to both the kinetic and the inertial terms are given by Lysak and Song [2003]. The acceleration due to kinetic terms has been computed by Das and Ip [1992], whereas the computations of the electron acceleration made by Crary [1997] were made using the inertial correction only. In this latter case the parallel electric field associated with the Alfvén wave $\delta E_{\|}$can be approximated by Hess et al. [2007b; Lysak and Song, 2003] as follows:

$$
\delta E_{\|} \simeq \omega_{a} k_{\perp} \lambda_{e}^{2} \delta B
$$

where $\omega_{a}$ is the Alfvén gyrofrequency and $\delta B$ is the magnetic field perturbation associated with the wave. The computation of the energies acquired by the particles in the torus [Crary, 1997] was made under the assumption that the mechanism of energy transfer between the wave and the particle was a wave-particle collision, that is, by assuming that the electrons see the wave as a potential wall with which they collide, thus acquiring kinetic energy [Kletzing, 1994]. Crary [1997] computed the collision rate and the energy exchange between the wave and the particles, obtaining a theoretical distribution of the particles outside the torus. This distribution is bimodal with a first maximum around $700 \mathrm{eV}$ and a second one around $400 \mathrm{keV}$. The mean energy is about $75 \mathrm{keV}$.

[55] As these particles propagate along the magnetic field lines, their parallel velocity decreases and is transferred to their perpendicular velocity due to the adiabatic motion of the particles in the converging magnetic field. However, because their velocity near the torus is almost purely parallel, only the most energetic particles can reach the Jovian ionosphere before their parallel velocity goes to zero. Thus a sufficient flux of particles must reach the Jovian ionosphere to produce the auroral spots. At lower energy, a sufficient number of particles must be reflected by magnetic mirroring to be able to produce the radio emissions. Thus the Crary [1997] model seems to explain accurately the Io-Jupiter interaction observations.

[56] However, there are some issues with these models. They include only the inertial term [Crary, 1997] or the kinetic term [Das and Ip, 1992] in the Alfvén wave dispersion but not both. As shown by equation (7), the kinetic and inertial term have opposite effects on the wave phase velocity and can balance each other, making the phase velocity equal to the group velocity. The parallel electric field associated with the Alfvern wave is a consequence of the difference between the phase and group velocities; taking into account both the kinetic and the inertial effects may decrease the efficiency of the acceleration process proposed by Crary [1997] and Das and Ip [1992]. Taking both the kinetic and inertial term into account, Jones and $\mathrm{Su}$ [2008] showed that the parallel electric field in the torus is negligible $(0.01 \mathrm{mV} / \mathrm{m}$ at the maximum); they also showed that the kinetic effects dominate slightly in the torus and vanish outside, whereas the inertial effects become strong at high latitudes. Thus the mechanisms proposed by Crary [1997] and Das and Ip [1992] cannot generate a significant acceleration near the torus necessary to power the auroral emissions.

\subsection{Particle Acceleration at High Latitudes}

\subsubsection{Acceleration Process}

[57] The high-latitude parallel electric field, being attributable to the inertial term, can be approximated by equation (23) [Lysak and Song, 2003], where the perpendicular scale is proportional to the flux tube section $\left(k_{\perp} \propto B^{1 / 2}\right)$. The acceleration of the particles depends on the perpendicular scale of the Alfvén waves; that is, smaller wavelengths result in stronger acceleration. The parallel electric field profiles for the inertial Alfvén waves peak between $\sim 0.5$ and $\sim 1.5 \mathrm{R}_{\mathrm{J}}$ in altitude from the Jovian ionosphere [Lysak and Song, 2003; Jones and Su, 2008; Hess et al., 2007b] (Figure 8). This wide range of values may be explained by the choice of the planetary magnetic field model and of the ionospheric density model. The lower altitudes are obtained for a dipolar approximation of the Jovian electric field and a cold ionospheric plasma population [Lysak and Song, 2003; Jones and $\mathrm{Su}, 2008]$, whereas using a more detailed model of the magnetic field (the VIP4 model of [Connerney et al., 1998]) and an ionospheric plasma heated by the auroral activity (consistent with the results of Su et al. [2003]) gives higher altitudes [Hess et al., 2007b]. In the present paper we use a parallel electric field profile consistent with that of Hess et al. [2007b] (Figure 8).

[58] Acceleration by inertial Alfvén waves at high latitude has been observed on Earth [Chaston et al., 2000, 2002, 2005, 2007a, 2007b; Andersson and Ergun, 2006]. Recently, Su et al. [2008] related the terrestrial millisecond radio bursts to electron acceleration by Alfvén waves above the auroral regions. From the terrestrial example some have postulated that at least part of the electron acceleration is due to such high-latitude acceleration [Su et al., 2006; Ergun et al., 2006].

[59] Many processes proposed to model this acceleration involve the resonance of the inertial Alfvén waves with the electrons in the auroral regions [Chen et al., 2005; Watt and Rankin, 2008]. However, the situation is quite different at Jupiter. At the acceleration altitudes, the Alfvén phase velocity is much larger than the particle velocities (up to a few percent of the speed of light) for most of the wavelengths and thus can not accelerate the electrons through some resonant interaction except for small perpendicular wavelengths $\left(<0.001 R_{J}\right)$, which are not expected to provide a large part of the electron acceleration.

[60] Electron acceleration by Alfvén waves on Jupiter occurs because of the localization of the parallel electric field. Electrons can be accelerated where the parallel electric field is strong, escapes the strong parallel electric field region before the wave phase changes, and then is only weakly decelerated. This acceleration process assumes that the electrons are fast enough to escape the strong parallel electric field region before the reversal of the wave phase. If such were not the case, the electrons would be successively accelerated and decelerated with no resulting acceleration. For this reason, the electron distribution obtained from the acceleration by inertial Alfvén waves has an almost unperturbed core and an extended tail in the parallel direction. 


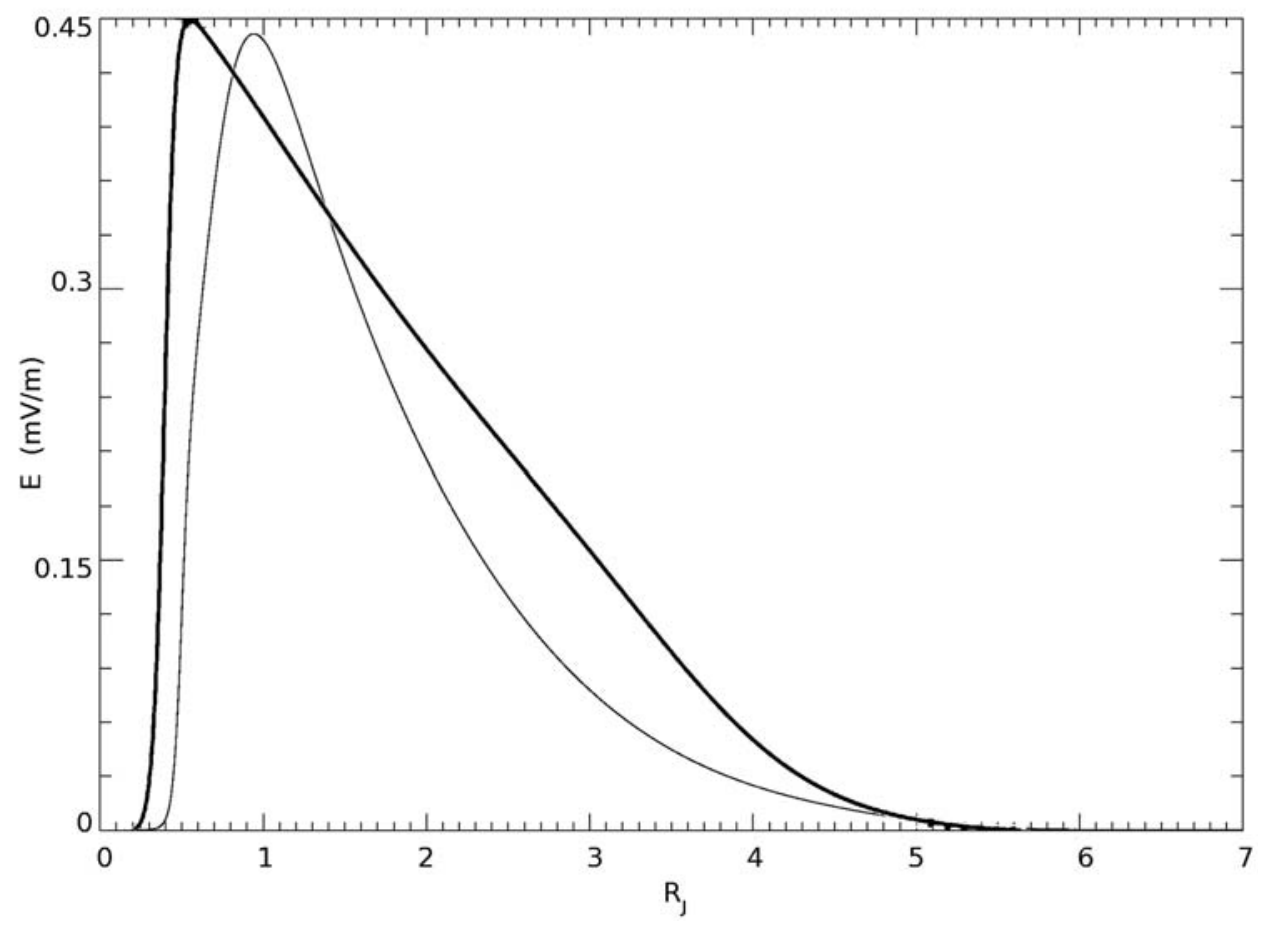

Figure 8. Parallel electric field associated with the Alfvén wave as a function of the altitude above the Jovian ionosphere. Bold line is adapted from Jones and $\mathrm{Su}$ [2008]; thin line is the one used in our study. The difference comes from the magnetic field model (Jones and Su [2008] used a dipolar field, whereas we used the VIP4 model for a magnetic field line longitude of 180 at the equator) and from the density profile (Jones and $S u$ [2008] assumed a cold ionosphere, whereas we assumed a heated one, consistent with simulations by Su et al. [2003]). We assumed a magnetic perturbation with an amplitude of $10 \mathrm{nT}$ and a perpendicular scale of $200 \mathrm{~km}$ at the equator.

Because the acceleration process is not dependent on the direction of propagation of the Alfvén wave, it can accelerate both toward Jupiter and in the opposite direction. This is well illustrated by Figures 9a and 9b, which show the simulated trajectories and the energies of several electrons (see next section for simulation details).

\subsubsection{Accelerated Distributions}

[61] The exact shape of the distribution may not be computed analytically, but it can be computed numerically. This has been done in two different ways by hybrid simulations using the Swift [2007] code and the work by Hess et al. [2007b]. The resulting distributions are shown in Figure 10.

[62] Swift [2007] used a hybrid numerical code to simulate the acceleration of the electron by an inertial Alfvén wave in the terrestrial auroral region. The parallel electric field profile in the simulation was similar to Jupiter's. We used the same numerical code to simulate the Io-Jupiter interaction. The electron population is assumed to consist of a hot magnetospheric component (temperature of $50 \mathrm{eV}$ ) and a cold, much denser ionospheric component. The assumed density of the hot component is given by

$$
n_{H}=n_{H ; 0} \tan h(z / \lambda)
$$

where $n_{H ; 0}=1 \mathrm{~cm}^{-3}, \lambda=4000 \mathrm{~km}$, and $z$ is the altitude above Jupiter, starting at the limit of its ionosphere, which is assumed to be parallel to the magnetic field. The cold component is assumed to vary as

$$
n_{C}=n_{C ; 0} \exp (-z / H)
$$

where $n_{C ; 0}=210^{5} \mathrm{~cm}^{-3}$ and $H=1142 \mathrm{~km}$. The electrons are strongly magnetized, so their only motion is parallel to the magnetic field direction. The ions are taken to be a nearly stationary fluid, which carries only a polarization current perpendicular to the magnetic field. A major difference between this model and the Swift [2007] simulation is that in this model, the densities are so low and the magnetic field is so strong that the nominal Alfvén velocity greatly exceeds the velocity of light. This necessitates the inclusion of the displacement current in the formulation of the model. The only significant change in the equations is that equation (6b) in Swift [2007] becomes

$$
\frac{\partial \phi}{\partial t}=c\left(\frac{v_{a}^{2}}{c^{2}+v_{a}^{2}}\right) \frac{1}{h_{2}} \frac{\partial}{\partial z}\left(h_{2} A_{\|}\right)
$$

where $\phi$ is the electrostatic potential of the wave, and $A_{\|}$is the parallel component of the magnetic vector potential. Here $h_{2}$ is a scale factor given by

$$
h_{2}=\left(\frac{z+R_{J}}{R_{J}}\right)^{1.5}
$$




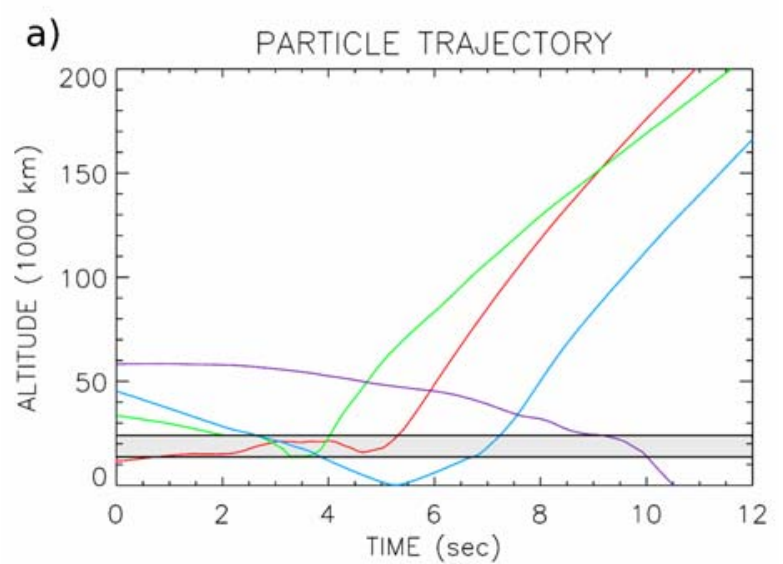

b)

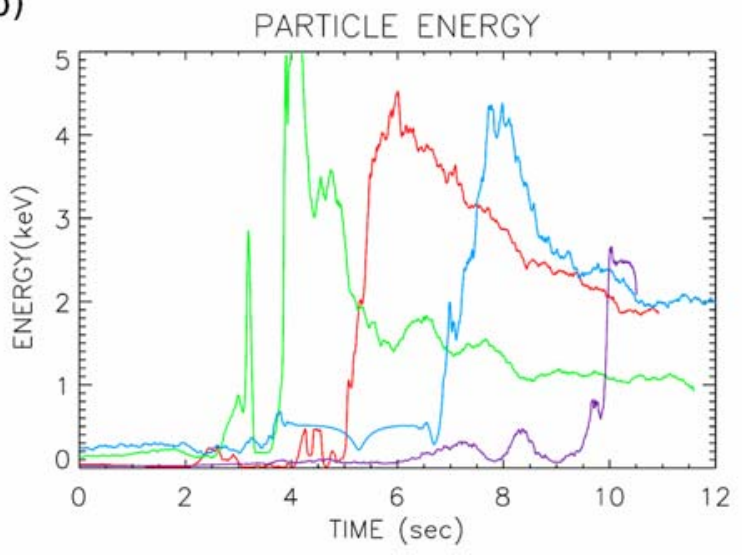

Figure 9. (a) Trajectories of four selected particles. The acceleration region, shown in gray, corresponds to $\sim 0.3$ $R_{J}$. The particles are accelerated both toward Jupiter and in the opposite direction. (b) Diagnostic of the kinetic energy of the same particles.

which takes into account the expansion of the magnetic flux tube in the direction perpendicular to the plane of the simulation. Because the scale factor perpendicular to the magnetic field in the plane of the simulation is the same, the flux tube cross section increases as the cube of the Joviocentric distance.

[63] The simulation domain extends from the top of the atmosphere to an altitude of $200,000 \mathrm{~km}$. The high-density plasma torus is not included in the simulation. The spatially narrow Alfvén wave pulse is launched by applying a variable potential with a peak amplitude of $\pm 200 \mathrm{~V}$ at the top of the simulation domain. The electron velocity distribution in the top boundary layer is biased to give a current consistent with a downward propagating Alfvén wave as described by Swift [2007]. With the bipolar excitation, the electric field of the incident and reflected waves reinforce each other to produce stronger electron acceleration.

[64] The resulting distribution is a Kappa-like function (the distribution tail is a power law) in the parallel direction (Figures 10a-10c). The average electron energy is a few $\mathrm{keV}$ both in Jupiter's direction and in the antiplanetward direction (Figure 10b). As the perpendicular scale of the wave decreases, the mean energy of the accelerated particles increases and the number of trapped particles decreases. This is due to the dependence of the parallel electric field on the
Alfvén wave perpendicular scale expressed by equation (23). As an obvious consequence the filamented waves are expected to provide a more efficient electron acceleration than the long-scale waves. Figure 9 shows the trajectories and the evolution of the kinetic energies of four particles in the simulation. The particles acquire few $\mathrm{keV}$ in a very small region, located at an altitude of $\sim 0.3 R_{J}$ above the Jovian ionosphere. This altitude corresponds roughly to the location of the parallel electric field peak in the simulation becausethe Swift [2007] hybrid code uses a dipolar field and cold ionospheric plasma assumption.

[65] Hess et al. [2007b] simulated the distribution of electrons along the Io-Jupiter magnetic field lines, assuming an acceleration by a monochromatic inertial Alfvén wave with an amplitude of $\sim 100 \mathrm{nT}$, which is much more that what one can expect for such a wave obtained from a filamentation process. However, such a monochromatic wave of large amplitude may be generated by the combination of a wavelength filtering by an ionospheric resonator [Su et al., 2006] and an amplification by ionospheric feedback [Lysak and Song, 2003]. Because the wave amplitude is large, most of the electrons are strongly accelerated and few are trapped. Thus the resulting distribution is a beam distribution and not a Kappa-like distribution (Figure 10d). The authors located the acceleration region around $0.8-0.9 R_{J}$ which corresponds to the location of the parallel electric field gradient in their simulation. By computing analytically the growth rate of the radio waves, Hess et al. [2007b] showed that such a wave may generate the Jovian millisecond radio bursts.

\subsubsection{Acceleration Efficiency}

[66] Although the exact shape of the distribution of the accelerated electrons cannot be computed analytically, the power transfer from the Alfvén wave to the particles can be estimated. The power of the wave through a cross section of the flux tube is given by the wave's Poynting flux:

$$
P_{w}=\frac{E \times B}{\mu_{0}} S=\frac{v_{\phi, a} \delta B^{2}}{\mu_{0}} S
$$

where $\delta B$ is the wave amplitude and $S$ the section of the flux tube. The power associated with the particles is obtained by multiplying the energy of the particles by the particle flux. The flux of downward particles through the cross section $S$ is conserved during the acceleration process and is given by the density $n$ times the thermal velocity $v_{t h}$. To compute the energy of the particle, we estimate it to be the energy acquired by the particle due to the parallel electric field of the wave during half a wave period:

$$
P_{e}=n v_{t h} \frac{m}{2}\left(\frac{\pi}{2} \frac{-e E_{\|}}{\omega_{a} m}\right)^{2} S=\frac{\pi^{2}}{8} \frac{\omega_{p}^{2}}{\omega_{a}^{2}} \epsilon_{0} v_{t h} E_{\|}^{2} S
$$

Using the expression of the parallel electric field given by equation (23), the power transmitted to the particles can be expressed as a function of the wave amplitude $\delta B$ and then as a function on the incident wave power $P_{w}$ using equation (28)

$$
\begin{aligned}
& P_{e}=\frac{\pi^{2}}{8} \omega_{p}^{2} \epsilon_{0} v_{t h} k_{\perp}^{2} \lambda_{e}^{4} \delta B^{2} S=\frac{\pi^{2}}{8} c^{2} \epsilon_{0} v_{t h} k_{\perp}^{2} \lambda_{e}^{2} \delta B^{2} S \\
& \frac{P_{e}}{P_{w}}=\frac{\pi^{2}}{8} \frac{v_{t h}}{v_{\phi, a}} k_{\perp}^{2} \lambda_{e}^{2} .
\end{aligned}
$$



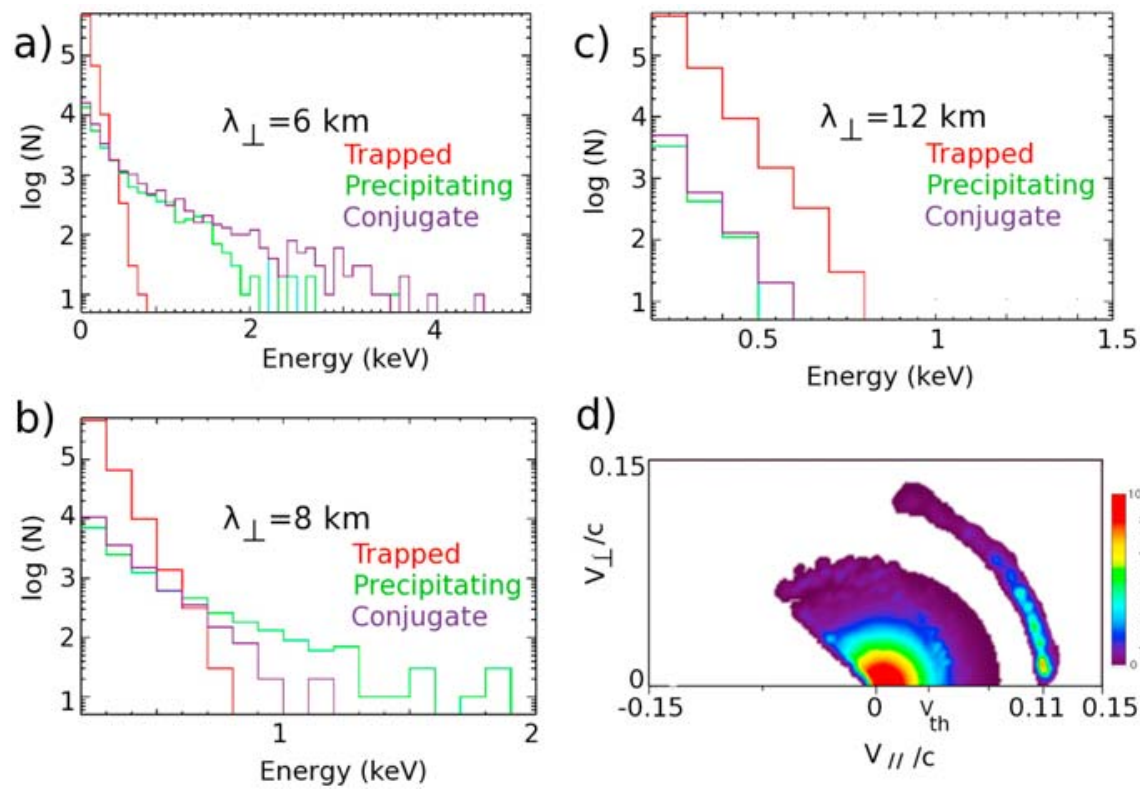

d)

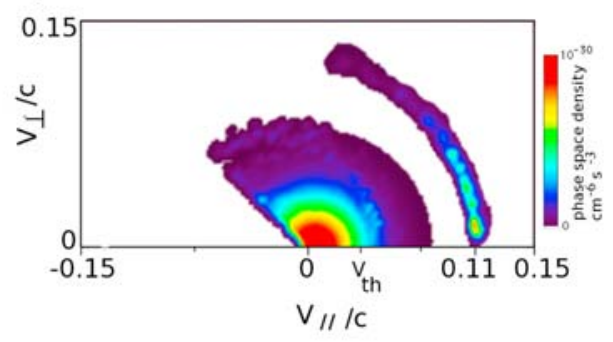

Figure 10. Histograms of the electron energy in the hybrid simulations (numerical code by Swift [2007] adapted to Jupiter's case): (a) $k_{\perp}=6 \mathrm{~km}$; (b) $k_{\perp}=8 \mathrm{~km}$; (c) $k_{\perp}=12 \mathrm{~km}$ at the top of the Jovian ionosphere. Electrons are accelerated toward Jupiter (precipitating) and backward (conjugate) with a power law distribution. A core of electrons does not precipitate (trapped). (d) Distribution function showing the beam of electrons resulting of the electron acceleration in Hess et al. [2007b].

This equation is given for a particular value of $\mathbf{k}$; to obtain the total power transfer, it is necessary to integrate over $\mathbf{k}$.

[67] We computed an estimate of the power transmission to the electrons for the three distributions previously defined. For the long-scale distribution, the power transmission to the particles is $0.05 \%$; for the filamented distributions $\left(f_{2}\right)$ it reaches $10 \%$, if a Kolmogorov $\left(k^{-\frac{5}{3}}\right)$ distribution can be assumed, and $4 \%$ for a $k^{-2}$ distribution. It is almost $100 \%$ for the short-scale distribution, even without taking into account that resonant interactions may exist for such short wavelengths.

[68] Assuming a power generated at Io of $10^{12} \mathrm{~W}$ and a filamentation by turbulent processes, we can determine the power transferred to the electrons, taking into account the transmission coefficient $(50 \%)$, as $2 \times 10^{10}-5 \times 10^{10} \mathrm{~W}$. The powers obtained for each distribution are summarized in Table 2. This power is divided between the particles moving toward and away from the planet (some of which may precipitate in the opposite hemisphere). Roughly $\sim 2 \times 10^{10} \mathrm{~W}$ are available to generate the emissions of the MAW spot (and of the MAW spot only, other spots being supplied by the reflected Alfvén wave power). Since the power of the precipitating particles in the MAW spot is estimated to be a few
$10^{10} \mathrm{~W}$ in UV, visible, IR, and radio, we fulfill the energy requirement.

[69] The $100 \%$ efficiency of the short-scale acceleration implies that the Alfvén wave packet is completely damped. Such damping was not taken into account in our estimation (except by imposing $\frac{P_{e}}{P_{e}} \leq 1$ ). However, these short waves may still be completely damped, thus transferring all their power to the electrons, in particular because the phase velocity is close to or lower than the electron thermal velocity for such short waves, and resonant interactions may occur. Our estimation adds the power transfer from each wavelength regardless of their phase, which may be an issue. However, for high electric potentials $\left(\gg \frac{m_{e}}{2} v_{e}^{2}\right)$, the Alfvén wave packet steepens toward an electric field spike (implying an in-phase acceleration of the electrons) [Hui and Seyler, 1992; Clark and Seyler, 1999; Swift, 2007]. These spiky structures of the electric field are responsible for the sharp accelerations seen in Figure 9.

\subsection{Additional Acceleration: Electric Potential Structures}

[70] Electric potential structures have long been observed in the terrestrial auroral region [Mozer et al., 1980; Carlson

Table 2. Summary of the Power Transmission Through the Torus and of the Efficiency of the Power Transmission to the Particles for Different Distributions of the Alfvén Wave Wavelengths

\begin{tabular}{lccc}
\hline Distribution Power at Io: $10^{12} \mathrm{~W}$ & Power Escaping the Torus ${ }^{\mathrm{a}}$ & $\begin{array}{c}\text { Efficiency of the Power } \\
\text { Transmission to Particles }\end{array}$ & $\begin{array}{c}\text { Power Transmitted } \\
\text { to the Particles }\end{array}$ \\
\hline Long scales & $17 \%$ & $0.05 \%$ & $\sim 10^{8} \mathrm{~W}$ \\
Filamented (power law) & $\sim 50 \%$ & $4-10 \%$ & $\sim 10^{10} \mathrm{~W}$ \\
Filamented (Compressional) & $90 \%$ & $100 \%$ & $\sim 10^{12} \mathrm{~W}$ \\
\hline
\end{tabular}

${ }^{\mathrm{a}}$ Power reaching $1 R_{J}$ above the Jovian ionosphere. 
et al., 1998; Andersson et al., 2002a, 2002b; Andersson and Ergun, 2006]. The existence of electric potential structures in the auroral region above the Io footprint was first observed by Hess et al. [2007a]. Those authors observed abrupt variations of the parallel velocity of the electrons responsible for the millisecond radio burst emissions, which are fully consistent with potential jumps. Using continuous observations over more than $1 \mathrm{~h}$, Hess et al. [2009b] showed that these potential drops are moving away from Jupiter at the local speed of sound and are generated near the ionospheric boundary with a quasi-period of $200 \mathrm{~s}$. Variations of the UV emissions with similar timescales have been observed by Bonfond et al. [2007] for the UV Io footprint and by Grodent et al. [2009] for the UV Ganymede footprint. Additionally, by studying local perturbations of the drift in frequency of the millisecond radio bursts Hess et al. [2009a] deduced the existence of bipolar potential structures. However, as pointed out by the latter authors, these electric potential structures, which provide an energy drop of a few hundred $\mathrm{eV}$ to the electrons, are not the primary source of acceleration, since the measured electron energy above the energy drops is equal to or larger than their cumulated amplitudes.

[71] A short, but complete, review of the origin of such potential structures can be found in the work of Chaston et al. [2007a]. These physical processes involve an intensification of the parallel electric current due to the Alfvén waves interaction with other waves or with density cavities. If the current reaches a given threshold [Block, 1988], it can lead to the formation of strong potential drops and may explain the observations.

\section{Discussion}

\subsection{Power Balance}

[72] Comparing the power emitted (from UV to lowfrequency radio) in the auroral features related to Io with theoretical models of the power generated at Io gives a consistent scheme concerning the amplitude and the phase of variations with respect to the longitude of Io. It is particularly true in the UV which has the most complete longitudinal coverage. The brightness curve of the MAW spot is highly correlated to the predicted power profile.

[73] The total precipitated power for the MAW spot from $\mathrm{UV}$ to radio ranges from a few $10^{9}$ to $10^{10} \mathrm{~W}$ (Table 1$)$. We compare this with the $10^{12} \mathrm{~W}$ that models give for the total power generated at Io [Neubauer, 1980; Goertz, 1983; Saur, 2004]. The comparison reveals that the efficiency of the power transfer from the local magnetic field perturbation in the Io torus to the auroral emissions must be relatively high (more than 1\%). The models describing the emission processes prescribe a theoretical efficiency of the power transfer from the precipitating particles to the radiation of nearly $10 \%$. This implies that several percent (up to $10 \%$ ) of the power generated at Io is converted into the kinetic energy of the precipitating particles. This puts very hard constraints on the processes transmitting the power from Io to Jupiter.

[74] The long-scale waves cannot accelerate particles in the vicinity of Jupiter since the parallel electric field they produce is too weak to transfer a large amount of the Alfvén wave power to the particles. Although several models predicted strong enough acceleration in the torus by these waves,
Jones and Su [2008], taking into account both the kinetic and inertial terms, showed that these mechanisms are ineffective.

[75] From a power balance point of view, the Io-Jupiter interaction likely involves filamented waves, which accelerate particles in Jupiter's vicinity. When comparing the power transmitted to the particles predicted by the model (a few $10^{10} \mathrm{~W}$ ) to the amount of power required by the UV to radio emissions (a few $10^{10} \mathrm{~W}$ ) we find a good agreement (Table 1).

\subsection{Particle Energy and Distribution}

[76] When dividing the $\sim 10^{10} \mathrm{~W}$ tranferred to the electrons (calculated in section 5.2) between each particle (we assume a hot electron density of $1 \mathrm{~cm}^{-3}$ ), a mean electron energy of $\sim 1 \mathrm{keV}$ is found. This value is consistent with the results of the simulations of Swift [2007] and Hess et al. [2007b], as well as with the value deduced from observations by Bonfond et al. [2009] (provided that their results on the tail can be applied to the main spot as suggested by the similar color ratios). Thus the high-latitude acceleration scheme gives results consistent with the observations.

[77] Concerning the distribution of the particles, the numerical simulations of Swift [2007] predict a Kappa-like distribution, consistent with the distribution of the electrons deduced by Bonfond et al. [2009] (same remark as above). On the other hand, the simulations of Hess et al. [2007b] predict the formation of a beam with an occurrence period equal to that of the Alfvén wave responsible for particle acceleration and also for reproducing the dynamic spectra of the radio short bursts.

[78] The fact that two simulations give two different results, both of which are consistent with some parts of the observations, may appear paradoxical. But the difference between the simulations is mainly the result of the amplitude of the magnetic perturbations (Alfvén waves) introduced. The simulation of Swift [2007] uses an amplitude that is consistent with an Iogenic Alfvén wave filamented through a Kolmogorov cascade $(\sim 10 \mathrm{nT}$ for a parallel scale near the electron inertial length). The simulation of Hess et al. [2007b] uses an amplitude 10 times larger ( 100 nT). Such large-amplitude, quasi-monochromatic Alfvén waves can be generated by the combination of both an ionospheric resonator and an ionospheric feedback [Lysak and Song, 2003]. As shown by Su et al. [2006], the ionospheric resonator may select large-amplitude modes with frequencies consistent with the occurrence frequency of the radio short bursts. Thus the two accelerated distributions modeled by Swift [2007] and Hess et al. [2007b] may coexist and generate different parts of the auroral emissions.

[79] Moreover, one should note that if we are able to observe modulations of the radio emissions with a timescale of few tens of milliseconds, we are not able to do the same for the UV to IR part of the emissions, partly because of the longer lifetime of the excited states of the hydrogen molecules and ions. Thus short bursts in the UV to IR range may exist but are not observable.

[80] Finally, the last argument for the existence of an acceleration region around $1 R_{J}$ above the ionosphere is that the short radio bursts imply a very low pitch angle dispersion between the acceleration region and the emission region. This is not possible if the acceleration occurs near the plasma torus edges. 


\subsection{Antiplanetward Acceleration}

[81] The Galileo measurements of the velocity distribution of electrons in the torus showed the presence of energetic beams of electrons [Frank and Paterson, 1999; Mauk et al., 2001]. From the beams' characteristics the authors deduced that they are accelerated at high latitudes (near Jupiter). Moreover, from the variation of position relative to the MAW UV spot of the TEB UV auroral spot, Bonfond et al. [2008] showed that the latter spot may be related to electron beams accelerated in the auroral regions of the opposite hemisphere. Thus any acceleration process that tries to describe the acceleration of the electrons at Jupiter must be able to predict an antiplanetward acceleration at high latitudes. The precipitated power on TEBs is estimated to be $\sim 10^{10} \mathrm{~W}$ [Bonfond et al., 2008].

[82] The acceleration due to the strong gradient of the parallel electric field near $1 R_{J}$ above the Jovian ionosphere generates such antiplanetward acceleration. This is confirmed by both the Swift [2007] and Hess et al. [2007b] models. The antiplanetward power is found to be $\sim 10^{10} \mathrm{~W}$ in our computation. The observations of such a beam are strong evidence that the acceleration occurs at high latitude as a result of this electric field gradient.

\section{Conclusion: Io-Jupiter Interaction Model}

[83] The energy source for Io-generated auroral emissions at Jupiter is provided by the motion of Io relative to the magnetic field of Jupiter and the frozen-in plasma torus. Both Io's motion relative to the magnetic field and the plasma flow perturbations generate an electric field, inducing a current that ultimately appears as a perturbation of the magnetic field lines. This perturbation propagates as Alfvén waves that carry the current along the magnetic field lines toward Jupiter. In the torus the Alfvén waves are likely filamented, acquiring shorter wavelengths; $\sim 50 \%$ of the Alfvén wave power is transmitted through the torus and reaches high latitudes. Owing to the filamentation, the Alfvén waves reaching high latitudes have a component with small perpendicular scales.

[84] These short-scale waves are able to generate a parallel electric field at $\sim 1 R_{J}$ above Jupiter's ionosphere. The acceleration resulting from the parallel electric field leads to a Kappa distribution of the electrons for the parallel velocities, with a mean electron energy of $\sim 1 \mathrm{keV}$. Additional nonlinear effects, such as Alfvén ionospheric resonance coupled to the ionospheric feedback, may amplify some frequencies leading to electron beams of a few $\mathrm{keV}$ whose occurrence are modulated at the amplified frequency. The power transmitted from the wave to the electrons reaches some $10^{10} \mathrm{~W}$. The accelerated electrons generate the UV, visible, and IR MAW spot emissions and the radio emissions (both emitting a few $10^{9} \mathrm{~W}$ ): a radio arc (for the Kappa-distribution) and the short radio bursts (for the electron beams). Moreover, the interaction between the Alfvén waves and the ionosphere may lead to the generation of electric potential drops which move along the field lines at the speed of sound and add some hundreds of $\mathrm{eV}$ to the precipitating electron energy.

[85] The acceleration resulting from the strong parallel electric field gradient accelerates electron beams both toward and away from Jupiter. This effect has been observed in the numerical simulations [Swift, 2007; Hess et al., 2007b]. These antiplanetward electrons may generate the electron beams observed in the torus by the Galileo probe and precipitate in the opposite hemisphere generating the transhemispheric spot in UV.

[86] The remaining 50\% of the Alfvén wave power, which has been reflected before the acceleration region, will act the same way as the primary Alfvén wave packet did; a part will escape, accelerate particles, and generate auroral emissions. These reflected waves are then responsible for the RAW spots. As the reflection does not occur at a given point but along an extended region, the reflected waves are able to interfere, the interference pattern becoming more and more complex at each reflection. This leads to the formation of a steady state current system in the wake of Io. The tail emission is thought to be the consequence of this current system [Ergun et al., 2009; Hill and Vasyliūnas, 2002].

[87] Figure 4 shows a sketch of the morphology of the Io-controlled aurorae at Jupiter predicted by the filamented waves model and compares them with the observed auroral emissions in UV, IR, and radio. The Galileo electron beam measurements are also indicated. This illustrates the correspondence between our model predictions and the observations.

[88] We have elucidated a general model for the transmission of the power from Io to Jupiter that gives some context to the detailed study of wave propagation and particle acceleration occurring between Io and Jupiter. This model implies a filamentation of the Alfvén wave packet in the vicinity of Io. For filamentation to happen fast enough, i.e., before the wave packet reaches the torus boundaries, nonlinear processes must be involved. This filamentation remains to be studied in detail. The model we propose can be used as a basis for more detailed investigations of electron acceleration by inertial Alfvén waves and can be used as a basis to study the interactions of other satellites (Europa, Ganymede, or Enceladus, for example).

[89] Acknowledgments. The authors thank F. Bagenal, J. Saur, and L. Ray for their valuable comments and suggestions. This work was funded by the NASA/GEOSPACE NNX07AF30G grants.

[90]. Wolfgang Baumjohann thanks the reviewers for their assistance in evaluating this paper.

\section{References}

Acuna, M. H., F. M. Neubauer, and N. F. Ness (1981), Standing Alfven wave current system at Io-Voyager 1 observations, J. Geophys. Res. 86, 8513-8521, doi:10.1029/JA086iA10p08513.

Alexandrova, O., et al. (2004), Cluster observations of finite amplitude Alfvén waves and small-scale magnetic filaments downstream of a quasi-perpendicular shock, J. Geophys. Res., 109, A05207, doi:10.1029/ 2003JA010056.

Andersson, L., and R. E. Ergun (2006), Acceleration of antiearthward electron fluxes in the auroral region, J. Geophys. Res., 111, A07203, doi:10.1029/2005JA011261.

Andersson, L., R. E. Ergun, D. L. Newman, J. P. McFadden, C. W. Carlson, and Y.-J. Su (2002a), Characteristics of parallel electric fields in the downward current region of the aurora, Phys. Plasmas, 9, 3600-3609, doi:10.1063/1.1490134

Andersson, L., N. Ivchenko, J. Clemmons, A. A. Namgaladze, B. Gustavsson, J.-E. Wahlund, L. Eliasson, and R. Y. Yurik (2002b), Electron signatures and Alfvén waves, J. Geophys. Res., 107(A9), 1244, doi:10.1029/ 2001JA900096.

Bagenal, F. (1994), Empirical model of the Io plasma torus: Voyager measurements, J. Geophys. Res., 99, 11,043-11,062, doi:10.1029/93JA02908.

Bigg, E. K. (1964), Influence of the satellite Io on Jupiter's decametric emission, Nature, 203, 1008-1010.

Block, L. P. (1988), Acceleration of auroral particles by magnetic-field aligned electric fields, Astrophys. Space Sci., 144, 135-147. 
Bonfond, B., J.-C. Gérard, D. Grodent, and J. Saur (2007), Ultraviolet Io footprint short timescale dynamics, Geophys. Res. Lett., 34, L06201, doi:10.1029/2006GL028765.

Bonfond, B., D. Grodent, J.-C. Gérard, A. Radioti, J. Saur, and S. Jacobsen (2008), UV Io footprint leading spot: A key feature for understanding the UV Io footprint multiplicity?, Geophys. Res. Lett., 35, L05107, doi:10.1029/2007GL032418.

Bonfond, B., D. Grodent, J.-C. Gérard, A. Radioti, V. Dols, P. A. Delamere, and J. T. Clarke (2009), The Io UV footprint: Location, inter-spot distances and tail vertical extent, J. Geophys. Res., 114, A07224, doi:10.1029/ 2009JA014312.

Burke, B. F., and K. L. Franklin (1955), Observations of a variable radio source associated with the planet Jupiter, J. Geophys. Res., 60, 213-217.

Carlson, C. W., et al. (1998), FAST observations in the downward auroral current region: Energetic upgoing electron beams, parallel potential drops, and ion heating, Geophys. Res. Lett., 25, 2017-2020, doi:10.1029/98GL00851.

Champeaux, S., T. Passot, and P. L. Sulem (1997), Alfvén-wave filamentation, J. Plasma Phys., 58, 665-690, doi:10.1017/S0022377897006119.

Champeaux, S., T. Passot, and P. L. Sulem (1998), Transverse collapse of Alfvén wave-trains with small dispersion, Phys. Plasmas, 5, 100-111, doi: $10.1063 / 1.872678$

Chaston, C. C., C. W. Carlson, R. E. Ergun, and J. P. McFadden (2000), Alfvén waves, density cavities and electron acceleration observed from the FAST spacecraft, Phys. Scr. T, 84, 64-68, doi:10.1238/Physica.Topical 084a00064.

Chaston, C. C., J. W. Bonnell, L. M. Peticolas, C. W. Carlson, J. P. McFadden, and R. E. Ergun (2002), Driven Alfven waves and electron acceleration: A FAST case study, Geophys. Res. Lett., 29(11), 1535, doi:10.1029/ 2001 GL013842.

Chaston, C. C., et al. (2005), Drift-kinetic Alfvén waves observed near a reconnection X line in the Earth's magnetopause, Phys. Rev. Lett., 95(6), 065002, doi:10.1103/PhysRevLett.95.065002.

Chaston, C. C., A. J. Hull, J. W. Bonnell, C. W. Carlson, R. E. Ergun, R. J. Strangeway, and J. P. McFadden (2007a), Large parallel electric fields, currents, and density cavities in dispersive Alfvén waves above the aurora, J. Geophys. Res., 112, A05215, doi:10.1029/2006JA012007.

Chaston, C. C., C. W. Carlson, J. P. McFadden, R. E. Ergun, and R. J. Strangeway (2007b), How important are dispersive Alfvén waves for auroral particle acceleration?, Geophys. Res. Lett., 34, L07101, doi:10.1029/2006GL029144.

Chen, L.-J., C. A. Kletzing, S. Hu, and S. R. Bounds (2005), Auroral electron dispersion below inverted-V energies: Resonant deceleration and acceleration by Alfvén waves, J. Geophys. Res., 110, A10S13, doi:10.1029/2005JA011168.

Chust, T., A. Roux, W. S. Kurth, D. A. Gurnett, M. G. Kivelson, and K. K. Khurana (2005), Are Io's Alfvén wings filamented? Galileo observations, Planet. Space Sci., 53, 395-412, doi:10.1016/j.pss.2004.09.021.

Clark, A. E., and C. E. Seyler (1999), Electron beam formation by small-scale oblique inertial Alfvén waves, J. Geophys. Res., 104 17,233-17,250, doi:10.1029/1999JA900212.

Clarke, J. T., et al. (1996), Far-ultraviolet imaging of Jupiter's aurora and the lo "footprint", Science, 274, 404-409, doi:10.1126/science. 274.5286.404.

Clarke, J. T., L. Ben Jaffel, and J.-C. Gérard (1998), Hubble Space Telescope imaging of Jupiter's UV aurora during the Galileo orbiter mission, J. Geophys. Res., 103, 20,217-20,236, doi:10.1029/98JE01130.

Clarke, J. T., et al. (2002), Ultraviolet emissions from the magnetic footprints of Io, Ganymede and Europa on Jupiter, Nature, 415, 997-1000.

Connerney, J. E. P., and T. Satoh (2000), The $\mathrm{H}_{3}^{+}$ion: A remote diagnostic of the Jovian magnetosphere, Philos. Trans. R. Soc. London, Ser. A, 358, 2471-2483.

Connerney, J. E. P., R. Baron, T. Satoh, and T. Owen (1993), Images of excited $\mathrm{H}_{3}^{+}$at the foot of the Io flux tube in Jupiter's atmosphere, Science, 262, 1035-1038, doi:10.1126/science.262.5136.1035.

Connerney, J. E. P., M. H. Acuña, N. F. Ness, and T. Satoh (1998), New models of Jupiter's magnetic field constrained by the Io flux tube footprint, J. Geophys. Res., 103, 11,929-11,940, doi:10.1029/97JA03726.

Crary, F. J. (1997), On the generation of an electron beam by Io, J. Geophys. Res., 102, 37-50, doi:10.1029/96JA02409.

Das, A. C., and W.-H. Ip (1992), Particle acceleration by kinetic Alfven waves in the Io plasma torus, Planet. Space Sci., 40, 1499-1502, doi:10.1016/0032-0633(92)90046-Q.

Delamere, P. A., F. Bagenal, R. Ergun, and Y.-J. Su (2003), Momentum transfer between the Io plasma wake and Jupiter's ionosphere, J. Geophys. Res., 108(A6), 1241, doi:10.1029/2002JA009530.

Dols, V., J. C. Gérard, J. T. Clarke, J. Gustin, and D. Grodent (2000), Diagnostics of the Jovian aurora deduced from ultraviolet spectroscopy:
Model and HST/GHRS observations, Icarus, 147, 251-266, doi:10.1006/ icar.2000.6415.

Dols, V., P. A. Delamere, and F. Bagenal (2008), A multispecies chemistry model of Io's local interaction with the Plasma Torus, J. Geophys. Res. 113, A09208, doi:10.1029/2007JA012805.

Ergun, R. E., Y.-J. Su, L. Andersson, F. Bagenal, P. A. Delemere, R. L. Lysak, and R. J. Strangeway (2006), S bursts and the Jupiter ionospheric Alfvén resonator, J. Geophys. Res., 111, A06212, doi:10.1029/ 2005JA011253.

Ergun, R. E., L. Ray, P. A. Delamere, F. Bagenal, V. Dols, and Y. J. Su (2009), Generation of parallel electric fields in the Jupiter-Io torus wake region, J. Geophys. Res., 114, A05201, doi:10.1029/2008JA013968.

Frank, L. A., and W. R. Paterson (1999), Intense electron beams observed at Io with the Galileo spacecraft, J. Geophys. Res., 104, 28,657-28,669. Galtier, S. (2009), Wave turbulence in magnetized plasmas, Nonlinear Proc. Geophys., 16, 83-98.

Galtier, S., S. V. Nazarenko, A. C. Newell, and A. Pouquet (2000), A weak turbulence theory for incompressible magnetohydrodynamics, J. Plasma Phys., 63, 447-488, doi:10.1017/S0022377899008284.

Gérard, J.-C., J. Gustin, D. Grodent, P. Delamere, and J. T. Clarke (2002), Excitation of the FUV Io tail on Jupiter: Characterization of the electron precipitation, J. Geophys. Res., 107(A11), 1394, doi:10.1029/ 2002JA009410.

Gérard, J.-C., A. Saglam, D. Grodent, and J. T. Clarke (2006), Morphology of the ultraviolet Io footprint emission and its control by Io's location, J. Geophys. Res., 111, A04202, doi:10.1029/2005JA011327.

Goertz, C. K. (1983), The Io-control of Jupiter's decametric radiation - The Alfven wave model, Adv. Space Res., 3, 59-70, doi:10.1016/0273-1177 (83)90257-0.

Grodent, D., B. Bonfond, A. Radioti, J.-C. Gérard, X. Jia, J. D. Nichols, and J. T. Clarke (2009), Auroral footprint of Ganymede, J. Geophys Res., 114, A07212, doi:10.1029/2009JA014289.

Gurnett, D. A., and C. K. Goertz (1981), Multiple Alfven wave reflections excited by Io origin of the Jovian decametric arcs, J. Geophys. Res., 86, 717-722, doi:10.1029/JA086iA02p00717.

Gurnett, D. A., W. S. Kurth, A. Roux, S. J. Bolton, and C. F. Kennel (1996), Galileo plasma wave observations in the Io plasma torus and near Io, Science, 274, 391-392.

Hess, S. (2008), Processus d'accélération et émissions radio dans le circuit Io-Jupiter, Ph.D. thesis, Univ. Pierre et Marie Curie, Paris.

Hess, S., P. Zarka, and F. Mottez (2007a), Io-Jupiter interaction, millisecond bursts and field-aligned potentials, Planet. Space Sci., 55, 89-99, doi:10.1016/j.pss.2006.05.016.

Hess, S., F. Mottez, and P. Zarka (2007b), Jovian S burst generation by Alfvén waves, J. Geophys. Res., 112, A11212, doi:10.1029/2006JA012191.

Hess, S., B. Cecconi, and P. Zarka (2008), Modeling of Io-Jupiter decameter arcs, emission beaming and energy source, Geophys. Res. Lett., 35 , L13107, doi:10.1029/2008GL033656.

Hess, S., F. Mottez, and P. Zarka (2009a), Effect of electric potential structures on Jovian S-burst morphology, Geophys. Res. Lett., 36, L14101, doi:10.1029/2009GL039084.

Hess, S., P. Zarka, F. Mottez, and V. B. Ryabov (2009b), Electric potential jumps in the Io-Jupiter flux tube, Planet. Space Sci., 57, 23-33, doi:10.1016/j.pss.2008.10.006.

Hess, S., A. Petin, P. Zarka, B. Cecconi, and B. Bonfond (2010), Modeling the Io-controlled radio-arcs: Lead angle and particle velocity measurements, Planet. Space Sci., in press.

Hill, T. W., and V. M. Vasyliūnas (2002), Jovian auroral signature of Io's corotational wake, J. Geophys. Res., 107(A12), 1464, doi:10.1029/ 2002JA009514

Hui, C., and C. E. Seyler (1992), Electron acceleration by Alfven waves in the magnetosphere, J. Geophys. Res., 97, 3953-3963, doi:10.1029/ 91JA03101.

Ingersoll, A. P., A. R. Vasavada, B. Little, C. D. Anger, S. J. Bolton, C. Alexander, K. P. Klaasen, and W. K. Tobiska (1998), Imaging Jupiter's aurora at visible wavelengths, Icarus, 135, 251-264, doi:10.1006/ icar.1998.5971.

Jacobsen, S., F. M. Neubauer, J. Saur, and N. Schilling (2007), Io's nonlinear MHD-wave field in the heterogeneous Jovian magnetosphere, Geophys. Res. Lett., 34, L10202, doi:10.1029/2006GL029187.

Jones, S. T., and Y.-J. Su (2008), Role of dispersive Alfvén waves in generating parallel electric fields along the Io-Jupiter fluxtube, J. Geophys. Res., 113, A12205, doi:10.1029/2008JA013512.

Kivelson, M. G., K. K. Khurana, R. J. Walker, J. Warnecke, C. T. Russell, J. A. Linker, D. J. Southwood, and C. Polanskey (1996), Io's interaction with the plasma torus: Galileo magnetometer report, Science, 274, 396-398.

Kletzing, C. A. (1994), Electron acceleration by kinetic Alfvén waves, J. Geophys. Res., 99, 11,095-11,104, doi:10.1029/94JA00345. 
Lecacheux, A., M. Y. Boudjada, H. O. Rucker, J. L. Bougeret, R. Manning, and M. L. Kaiser (1998), Jovian decameter emissions observed by the Wind/WAVES radioastronomy experiment, Astron. Astrophys., 329 , 776-784.

Louarn, P., J. E. Wahlund, T. Chust, H. de Feraudy, A. Roux, B. Holback, P. O. Dovner, A. I. Eriksson, and G. Holmgren (1994), Observation of kinetic Alfvén waves by the FREJA spacecraft, Geophys. Res. Lett., 21, 1847-1850, doi:10.1029/94GL00882.

Lysak, R. L., and Y. Song (2003), Kinetic theory of the Alfvén wave acceleration of auroral electrons, J. Geophys. Res., 108(A4), 8005, doi:10.1029/ 2002JA009406.

Mauk, B. H., D. J. Williams, and A. Eviatar (2001), Understanding Io's space environment interaction: Recent energetic electron measurements from Galileo, J. Geophys. Res., 106, 26,195-26,208, doi:10.1029/ 2000JA002508.

Moncuquet, M., F. Bagenal, and N. Meyer-Vernet (2002), Latitudinal structure of outer Io plasma torus, J. Geophys. Res., 107(A9), 1260 doi:10.1029/2001JA900124.

Mozer, F. S., C. A. Cattell, M. K. Hudson, R. L. Lysak, M. Temerin, and R. B. Torbert (1980), Satellite measurements and theories of low altitude auroral particle acceleration, Space Sci. Rev., 27, 155-213, doi:10.1007/ BF00212238.

Neubauer, F. M. (1980), Nonlinear standing Alfven wave current system at Io - Theory, J. Geophys. Res., 85, 1171-1178.

Prangé, R., D. Rego, D. Southwood, P. Zarka, S. Miller, and W. Ip (1996), Rapid energy dissipation and variability of the Io-Jupiter electrodynamic circuit, Nature, 379, 323-325, doi:10.1038/379323a0.

Prangé, R., D. Rego, L. Pallier, J. Connerney, P. Zarka, and J. Queinnec (1998), Detailed study of FUV Jovian auroral features with the postCOSTAR HST faint object camera, J. Geophys. Res., 103, 20,19520,216, doi:10.1029/98JE01128.

Pritchett, P. L., R. J. Strangeway, R. E. Ergun, and C. W. Carlson (2002), Generation and propagation of cyclotron maser emissions in the finite auroral kilometric radiation source cavity, J. Geophys. Res., 107(A12), 1437, doi:10.1029/2002JA009403.

Queinnec, J., and P. Zarka (1998), Io-controlled decameter arcs and Io-Jupiter interaction, J. Geophys. Res., 103, 26,649-26,666, doi:10.1029/ 98JA02435.

Queinnec, J., and P. Zarka (2001), Flux, power, energy and polarization of Jovian S-bursts, Planet. Space Sci., 49, 365-376.

Satoh, T., and J. E. P. Connerney (1999), Jupiter's $H_{3}^{+}$Emissions viewed in corrected Jovimagnetic coordinates, Icarus, 141, 236-252, doi:10.1006/ icar.1999.6173.

Saur, J. (2004) A model of Io's local electric field for a combined Alfvénic and unipolar inductor far-field coupling, J. Geophys. Res., 109, A01210, doi:10.1029/2002JA009354

Saur, J., F. M. Neubauer, D. F. Strobel, and M. E. Summers (1999), Threedimensional plasma simulation of Io's interaction with the Io plasma torus: Asymmetric plasma flow, J. Geophys. Res., 104, 25,105-25,126, doi:10.1029/1999JA900304

Saur, J., F. M. Neubauer, D. F. Strobel, and M. E. Summers (2002a), Interpretation of Galileo's Io plasma and field observations: I0, I24, and I27 flybys and close polar passes, J. Geophys. Res., 107(A12), 1422, doi:10.1029/2001JA005067.

Saur, J., H. Politano, A. Pouquet, and W. H. Matthaeus (2002b), Evidence for weak MHD turbulence in the middle magnetosphere of Jupiter, Astron. Astrophys., 386, 699-708, doi:10.1051/0004-6361:20020305.

Sharma, R. P., M. Malik, and H. D. Singh (2008a), Nonlinear theory of kinetic Alfvén waves propagation and multiple filament formation, Phys. Plasmas, 15(6), 062902, doi:10.1063/1.2927445.

Sharma, R. P., S. Kumar, and H. D. Singh (2008b), Nonlinear evolution of kinetic Alfvén waves and the turbulent spectra, Phys. Plasmas, 15(8), 082902, doi:10.1063/1.2970939.
Shukla, A., and R. P. Sharma (2002), Mutual nonlinear interaction between two kinetic Alfven waves and its effect on filamentation: Applications to solar wind and coronal heating, J. Geophys. Res., 107(A11), 1338, doi:10.1029/2001JA009135.

Shukla, P. K., and L. Stenflo (2000), Generation of localized density perturbations by shear Alfvén waves, Phys. Plasmas, 7, 2738-2739, doi:10.1063/1.874124.

Stasiewicz, K., G. Gustafsson, G. Marklund, P.-A. Lindqvist, J. Clemmons, and L. Zanetti (1997), Cavity resonators and Alfvén resonance cones observed on Freja, J. Geophys. Res., 102, 2565-2576, doi:10.1029/ 96JA03462.

Su, Y.-J., R. E. Ergun, F. Bagenal, and P. A. Delamere (2003), Io-related Jovian auroral arcs: Modeling parallel electric fields, J. Geophys. Res. 108(A2), 1094, doi:10.1029/2002JA009247.

Su, Y.-J., S. T. Jones, R. E. Ergun, F. Bagenal, S. E. Parker, P. A. Delamere, and R. L. Lysak (2006), Io-Jupiter interaction: Alfvén wave propagation and ionospheric Alfvén resonator, J. Geophys. Res., 111, A06211, doi:10.1029/2005JA011252.

Su, Y.-J., L. Ma, R. E. Ergun, P. L. Pritchett, and C. W. Carlson (2008), Short-burst auroral radiations in Alfvénic acceleration regions: FAST observations, J. Geophys. Res., 113, A08214, doi:10.1029/2007JA012896.

Swift, D. W. (2007), Simulation of auroral electron acceleration by inertial Alfvén waves, J. Geophys. Res., 112, A12207, doi:10.1029/2007JA012423.

Treumann, R. A. (2006), The electron cyclotron maser for astrophysical application, Astron. Astrophys. Rev., 13, 229-315, doi:10.1007/s00159006-0001-y.

Vasavada, A. R., A. H. Bouchez, A. P. Ingersoll, B. Little, C. D. Anger, and The Galileo SSI Team (1999), Jupiter's visible aurora and Io footprint, J. Geophys. Res., 104, 27,133-27,142, doi:10.1029/1999JE001055.

Watt, C. E. J., and R. Rankin (2008), Electron acceleration and parallel electric fields due to kinetic Alfvén waves in plasma with similar thermal and Alfvén speeds, Adv. Space Res., 42, 964-969, doi:10.1016/j.asr. 2007.03.030.

Williams, D. J., and R. M. Thorne (2003), Energetic particles over Io's polar caps, J. Geophys. Res., 108(A11), 1397, doi:10.1029/2003JA009980.

Williams, D. J., et al. (1996), Electron beams and ion composition measured at Io and in its torus, Science, 274, 401-403, doi:10.1126/science. 274.5286 .401

Williams, D. J., R. M. Thorne, and B. Mauk (1999), Energetic electron beams and trapped electrons at Io, J. Geophys. Res., 104, 14,739-14,754, doi:10.1029/1999JA900115.

Wright, A. N. (1987), The interaction of Io's Alfven waves with the Jovian magnetosphere, J. Geophys. Res., 92, 9963-9970, doi:10.1029/ JA092iA09p09963.

Yung, Y. L., G. R. Gladstone, K. M. Chang, J. M. Ajello, and S. K. Srivastava (1982), H2 fluorescence spectrum from 1200 to $1700 \mathrm{~A}$ by electron impact - Laboratory study and application to Jovian aurora, Astrophys. J., 254, L65-L69, doi:10.1086/183757.

Zarka, P. (1998), Auroral radio emissions at the outer planets: Observations and theories, J. Geophys. Res., 103, 20,159-20,194, doi:10.1029/ 98JE01323.

Zarka, P., T. Farges, B. P. Ryabov, M. Abada-Simon, and L. Denis (1996), A scenario for Jovian S-bursts, Geophys. Res. Lett., 23, 125-128, doi:10.1029/95GL03780.

B. Bonfond, Institut d'Astrophysique et Géophysique, Université de Liège, Place du 20, F-4000 Liège, Belgium.

P. Delamere, V. Dols, and S. L. G. Hess, Laboratory for Atmospheric and Space Physics, University of Colorado at Boulder, 1234 Innovation Dr., Boulder, CO 80303, USA. (sebastien.hess@obspm.fr)

D. Swift, Geophysical Institute, University of Alaska, Fairbanks, 903 Koyukuk Dr., Fairbanks, AK 99775, USA. 